\title{
9 Gestaltung, Implementation und Durchführungsevaluation des Unterrichts
}

In den vorangegangenen Kapiteln wurde beschrieben, dass die Nutzung elektronischer Medien im Alltag von Kindern und Jugendlichen bereits ab der Vorschulzeit eine bedeutende Rolle spielt (vlg. Kapitel 2), während sich die kognitiven und emotionalen Kompetenzen die zur Nutzung dieser Medien notwendig sind, erst im Laufe der Kindheit und frühen Adoleszenz entwickeln (vlg. Kapitel 3). Es wurde gezeigt, dass die Nutzung elektronischer Medien für Kinder und Jugendliche zahlreiche Funktionen bei der Entwicklung und Alttagsbewältigung erfüllen kann (vlg. Kapitel 3, S. 56 - 59), dass eine zeitlich exzessive oder inhaltlich problematische Mediennutzung aber auch negative Wirkungen auf schulische Leistungen, das Sozialverhalten, körperliches Wohlbefinden (Schlafstörungen, muskuloskeletale Beschwerden, Adipositats und Essstörungen) haben kann (vgl. Kapitel 4). Darüber hinaus wurde am Beispiel der Computerspielabhängigkeit gezeigt, wie problematische Mediennutzungsmuster mittelfristig zu Formen von Verhaltenssucht führen können (vgl. S. 115 - 124), durch die die beschrieben negativen Wirkungsmechanismen weiter verstärkt werden. Auf dieser Grundlage wurden problematische Mediennutzungsmuster bei Kindern und Jugendlichen definiert und in ihrer Prävalenz beschrieben (vlg. Kapitel 6), wobei für Vorschulkinder, Kinder und Jugendliche - soweit möglich kritische Merkmale zeitlicher, inhaltlicher und dysfunktionaler Mediennutzung erarbeitet wurden (vgl. die Zusammenfassung auf S. 153 - 154).

Anschließend wurde mithilfe der theoretischen Perspektiven Medienkompetenz und Health Promotion untersucht, mit welchem Instrumentarium die Entstehung problematischer Mediennutzungsmuster bereits im Kindesalter verhindert werden könnte (vgl. Kapitel 7), welche Voraussetzungen zu beachten sind und welche bereits etablierten Konzepte in Überlegungen zur Prävention medienbezogenen Risikoverhaltens im Kindesalter einbezogen werden können (vgl. Kapitel 8). Dabei wurde deutlich, dass insbesondere die Health-PromotionPerspektive ein Ansatz ist, welcher der vorher beschriebenen Problematik exzessiver Mediennutzungsmuster gerecht wird. So werden erstens Befunde der Medienwirkungsforschung von Vertretern dieser Perspektive ernst genommen, zweitens existiert durch die Arbeiten zur Primärprävention kindlichen und jugendlichen Risikoverhaltens bereits ein Instrumentarium, an das sich Konzepte der Verhinderung problematischer Mediennutzungsmuster anlehnen können. 
Schließlich wurde das Ziel formuliert, ein in der Praxis leicht anwendbares schulisches Primärpräventionskonzept für Grundschulkinder ab der dritten Klasse zu entwickeln, das von Grundschullehrkräften vermittelt wird und welches auch das familiäre Umfeld der Kinder einbezieht. Es wurde ferner der Anspruch formuliert, dass das Programm auch Kinder mit bereits bestehenden problematischen Mediennutzungsmustern ansprechen soll und dass der Erfolg des Programms nach wissenschaftlichen Kriterien bewertet werden soll. Das nun folgende Kapitel beschreibt die konkreten Schritte bei der Entwicklung und Durchführung dieses schulischen Präventionskonzeptes.

\subsection{Der Berliner Längsschnitt Medien - Zur Wirkung schulbasierter Unter- richtsinterventionen auf die kindliche Mediennutzung}

Um die Implementation medienerzieherischer Inhalte und die Effekte primärpräventiver Prävention problematischer Mediennutzung in der Grundschule möglichst langfristig messen zu können, wurde als Ort für die Implementierung und Evaluation des Präventionsprojektes das Bundesland Berlin ausgewählt, da hier der Regelbesuch der Grundschule bis zur sechsten Klasse vorgesehen ist. Die Schülerinnen und Schüler bleiben bis Ende der sechsten Klasse in ihrem Klassenverband und können so vier Jahre lang mithilfe der gleichen Methode im Klassenverband befragt werden. Zudem bietet das Land Berlin aufgrund seiner Bevölkerungsdichte, Geschichte und geografischen Lage die einmalige Chance, Schülerinnen und Schüler aus sehr unterschiedlichen sozialen und kulturellen Bezügen zu untersuchen.

Die Untersuchung mit dem Namen Berliner Längsschnitt Medien bildete den dritten sozialwissenschaftlichen Teil des von der Volkswagenstiftung finanzierten interdisziplinären und multizentrischen Forschungsprojektes Mediennutzung und Schulleistung (vgl. die Projektbeschreibung in der Einleitung dieser Arbeit ab S. 20). Es ist zu beachten, dass mithilfe des Datensatzes dieser Längsschnittuntersuchung zwei Forschungsfragen parallel untersucht wurden. Die nachfolgenden Ausführungen zum Berliner Längsschnitt Medien in der vorliegenden Arbeit beziehen sich ausschließlich auf den Interventionsteil der Untersuchung, in dem mithilfe eines Kontrollgruppendesign die längsschnittlichen Effekte von Medienunterricht in der Grundschule untersucht wurden (vgl. Abbildung 3, S. 236). Eine dazu parallel laufende Studie untersuchte - unabhängig von medienerzieherischen schulischen Aktivitäten - mithilfe der gleichen Daten langfristige Effekte kindlicher Mediennutzungsmuster auf schulische Leistung, Intelligenz, Sozialverhalten und körperliche Fitness von 
Schülerinnen und Schülern. Erste Ergebnisse dieses Studienteils wurden parallel zur vorliegenden Veröffentlichung von Mößle, Kleimann, Rehbein und Pfeiffer (2010) sowie Mößle und Roth (2009) publiziert.

Im Mai 2005 stimmte die Berliner Senatsverwaltung für Bildung, Jugend und Sport der Durchführung einer vierjährigen Unterrichtsevaluationsstudie zum Medienunterricht in Grundschulen zu. Durch die längsschnittliche Konzeption des Berliner Längsschnitt Medien eröffnete sich für das Präventionsprojekt die wichtige Möglichkeit, die Vermittlung der Präventionsinhalte nicht auf eine Unterrichtseinheit zu beschränken, sondern mit den Schülerinnen und Schülern im Rahmen mehrerer Unterrichtsinterventionen in drei aufeinanderfolgenden Jahren zum Thema der kindlichen Mediennutzung und ihren Risiken zu arbeiten und durch eine Befragung im darauf folgenden Jahr auch längerfristige Effekte medienerzieherischer Maßnahmen in der Schule zu ermitteln. Mit der Entscheidung für das Bundesland Berlin als Ort zur Durchführung und Evaluation des Medienunterrichtes ergaben sich auch bestimmte Voraussetzungen für die Möglichkeiten der Einbettung entsprechender Unterrichtsinhalte in die jeweiligen Rahmenlehrpläne des Bundeslandes Berlin, die in Kapitel 8 (ab S. 207) bereits beschrieben wurden.

Die Studie wurde als feldexperimentelles Kontrollgruppendesign angelegt (vgl. Abbildung 3, S. 236). Einer Gruppe zufällig ausgewählter Berliner Grundschulklassen, in denen in drei aufeinander folgenden Jahren Medienunterrichtseinheiten durchgeführt werden sollten, wurden 20 zufällig ausgewählte Berliner Grundschulklassen gegenübergestellt, in denen kein Medienunterricht stattfand. Die in Abbildung 3 dargestellte dritte Gruppe, die Baselinegruppe, spielt für die vorliegende Untersuchung zwar keine weitere Rolle, wird jedoch dennoch erwähnt, da sie Teil der ursprünglichen Stichprobe war (vgl. die Ausführungen auf S. 237 sowie Fußnote 152 


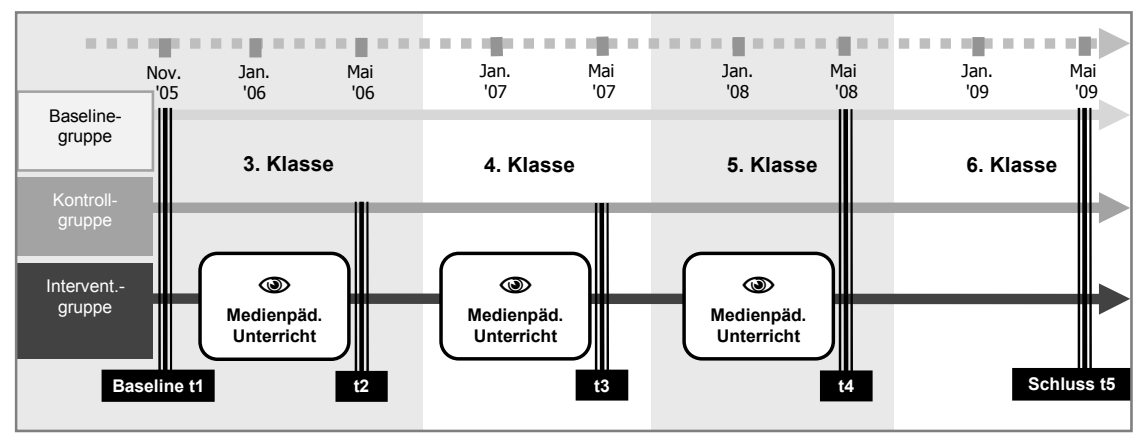

Abbildung 3: Studiendesign des Berliner Längsschnitt Medien

\subsubsection{Stichprobenziehung und Zuweisung zur Unterrichtsbedingung}

Aus der Grundgesamtheit aller $\mathrm{N}=1.042$ Berliner Klassen ${ }^{151}$ der dritten Jahrgangsstufe, in denen im Mai $2005 \mathrm{~N}=24.714$ Kinder beschult wurden, wurden zunächst jene Klassen ausgeschlossen, in denen sich $n=15$ oder weniger Schülerinnen und Schüler befanden. Die verbleibenden $n=1009$ Klassen mit $\mathrm{N}=24.352$ Schülerinnen und Schülern wurden in eine zufällige Stichprobenziehung aufgenommen. Anschließend wurden $n=80$ Klassen aus jeweils unterschiedlichen Grundschulen zufällig ausgewählt, wobei Ostberliner Klassen mit einer etwas höheren Gewichtung in die Ziehung eingingen als Westberliner Klassen. In einem Anschreiben an die Schulleitungen der gezogenen $n=80$ Klassen im Frühjahr 2005, welches von einem Empfehlungsschreiben Berliner Senats für Bildung, Jugend und Sport begleitet war, wurden die Schulleiter um Mitwirkung an der Studie gebeten (vgl. Anhänge A2 und A3). Wurde von der Schulleitung eine schriftliche Genehmigung zur Durchführung der Studie an ihrer Schule unterschrieben, wurden die Schulleiterinnen beziehungsweise Schulleiter gebeten, ein Schreiben mit der Bitte um Teilnahme an der Studie an die Klassenleitung der vorher gezogenen Klasse zu übergeben. Sowohl in den Direktorenanschreiben als auch in den Schreiben an die Klassenlehrkräfte (vgl. Anhang A4) wurde darauf hingewiesen, dass die Zuweisung der betreffenden Klasse zur Unterrichtsgruppe oder zur Kontrollgruppe noch nicht erfolgt sei und

151 Eine entsprechende Klassenliste wurde dem KFN vom Berliner Senat für Bildung, Jugend und Sport zur Verfügung gestellt. 
das Resultat einer Zufallsziehung sei. Letztlich erklärten sich $n=47$ Klassenlehrerinnen beziehungsweise Klassenlehrer grundsätzlich bereit, an der Studie teilzunehmen, sieben Lehrkräfte aus dieser Gruppe teilten allerdings im Vorhinein mit, aus schulorganisatorischen Gründen nicht am Unterrichtsteil der Studie teilnehmen zu können. Diese sieben Klassen wurden der so genannten Baseline-Bedingung zugewiesen, in deren Rahmen kein Medienunterricht stattfinden sollte und nur drei statt fünf Befragungen vorgenommen wurden ${ }^{152}$.

Im November 2005 wurden alle Schülerinnen und Schüler der 47 Klassen zu verschiedenen Lebensbereichen befragt, insbesondere ihrem schulischen und familiären Umfeld sowie ihrem Freizeitverhalten ${ }^{153}$. Von den $n=1.129$ in den Klassen beschulten Kindern nahmen $n=943$ Schülerinnen und Schüler, von denen eine elterliche Genehmigung zur Teilnahme an der Befragung vorlag, an der Untersuchung zum ersten Messzeitpunkt teil. Erst im Anschluss an diesen ersten Messzeitpunkt (im Folgenden abgekürzt mit MZP1) fand die zufallsbasierte Zuweisung der Klassen zur Unterrichtsbedingung beziehungsweise Kontrollbedingung statt, um aufgrund der t1-Messung der Mediennutzungszeiten und der Intelligenz der Schülerinnen und Schüler darüber befinden zu können, ob Schülerinnen und Schüler der Unterrichtsgruppe und der Kontrollgruppe bezüglich dieser Kriterien vergleichbar sind. Da sich hinsichtlich dieser Kriterien keine grundsätzlichen Bedenken zur Vergleichbarkeit der Gruppen ergaben, wurde den Lehrkräften Anfang Januar 2006 mitgeteilt, welcher Gruppe ihre Klasse zugeteilt wurde. 20 Klassen mit insgesamt $n=498$ Schülerinnen und Schülern wurden der Unterrichtsgruppe zugeteilt, 20 Klassen mit insgesamt $\mathrm{n}=469$ Schülerinnen und Schülern der Kontrollgruppe. Die Klassenlehrkräfte der Unterrichtsgruppe bekamen mit der Benachrichtigung über die Gruppenzuweisung gleichzeitig eine Einladung zum ersten Lehrkräfteworkshop Mitte Februar 2006, in dessen Rahmen Einzelheiten zur ersten Medienunterrichtseinheit mit ihnen erarbeitet werden sollten. Nach der Baseline-Messung im November 2005 (MZP1) fanden zwischen Mai und Juni 2006 (MZP2), 2007 (MZP3), 2008 (MZP4) und 2009 (MZP5) vier weitere Erhebungen mit dem in Tabelle 12 auf Seite 299 dieser Arbeit beschriebenen Instrumentarium statt. Im

152 Ursprünglich sollte ein Vergleich zwischen Baseline-Gruppe und Kontrollgruppe Aussagen darüber ermöglichen, inwieweit allein die alljährliche Messung der kindlichen Mediennutzung selbst ohne Medienunterricht einen interventionsähnlichen Effekt erzielt. Aufgrund der speziellen schulischen Bedingungen in den Baseline-Klassen, erwies sich ein Vergleich zwischen Kontrollgruppe und Baselinegruppe als zu artifiziell, um wissenschaftliche Aussagen zu dieser Frage machen zu können.

153 Eine ausführliche Übersicht zur den verwendeten Erhebungsinstrumenten findet sich in Tabelle 12 auf Seite 299 dieser Arbeit. 
Frühjahr 2006, 2007 und 2008 wurden in den Unterrichtsklassen die auf den Seiten 248 bis 293 beschriebenen Unterrichtseinheiten durchgeführt. Im Vorfeld dieser Unterrichtseinheiten fanden im Februar 2006, im Februar 2007 und im Februar 2008 jeweils Lehrkräfteworkshops zur Vorbereitung der Unterrichtseinheiten statt. Eine Übersicht über alle drei Unterrichtseinheiten, teilnehmende Klassenpopulationen sowie zentrale Instrumente dieser Unterrichtseinheiten finden sich in Tabelle 3 auf Seite 239. 
Tabelle 3: Unterrichtseinheiten des schulischen Medienlotsen-Präventionsprojektes

\begin{tabular}{|c|c|c|c|}
\hline Zielgruppe & $\begin{array}{c}\text { Vom Leichtmatrosen } \\
\text { zum Medienlotsen } \\
\text { (März - Mai 2006) }\end{array}$ & $\begin{array}{c}\text { Die ELIZA-Protokolle } \\
\text { (März - Mai 2007) }\end{array}$ & $\begin{array}{c}\text { Medienlotsen } \\
\text { gesucht! } \\
\text { (März - Mai } \\
\text { 2008) } \\
\end{array}$ \\
\hline Klassen & $n=19$ & $n=18$ & $n=5$ \\
\hline Schüler/innen & $\begin{array}{l}\qquad n=471 \\
\text { Lesegeschichte } \\
\text { Arbeitsblätter } \\
\text { Medientagebuch } \\
\text { Fernsehplan } \\
\text { Medienvertrag }\end{array}$ & $\begin{array}{l}\quad n=442 \\
\text { Lesegeschichte } \\
\text { Arbeitsblätter }\end{array}$ & $\begin{array}{c}n=111 \\
\text { Wettbewerb }\end{array}$ \\
\hline Eltern & $\begin{array}{c}\text { Elternabende } \\
\text { Dreisprachige } \\
\text { Informationsbroschüre } \\
\text { (Frühjahr 2006) }\end{array}$ & $\begin{array}{c}\text { DVDs }(12 / 2007) \\
\text { Dreisprachige } \\
\text { Informationsbroschüre } \\
(12 / 2007)\end{array}$ & \\
\hline Lehrer/innen & $\begin{array}{l}\text { Workshop } \\
(2 / 2006)\end{array}$ & $\begin{array}{l}\text { Workshop } \\
(2 / 2007)\end{array}$ & $\begin{array}{l}\text { Workshop } \\
(2 / 2008)\end{array}$ \\
\hline
\end{tabular}

Anmerkung: Die Zahl der in der Tabelle angegebenen Teilnehmerinnen und Teilnehmer weicht von der Zahl der auf Seite 237 angegebenen Schülerinnen und Schüler aller Unterrichtsklassen ab, da die Unterrichtseinheiten nicht in allen Klassen durchgeführt wurden.

\subsection{Expertenworkshop}

Am 12. Juli 2005 fand ein erster Expertenworkshop zur Entwicklung geeigneter Medienunterrichtsinhalte für Schülerinnen und Schüler ab der dritten Klasse statt. Zwei Mitglieder des KFN-Forschungsteams erörterten mit zwei Experten für Primarschuldidaktik und Medienpädagogik des Niedersächsischen Landesamtes für Lehrerbildung und Schulentwicklung (NiLS), einer Lehrerin für die Sekundarstufe sowie einem Autoren und Produzenten von Unterrichtsfilmen und Medieneinheiten Bedingungen, Inhalte und Didaktik einer Medienunterrichts- 
einheit. Moderiert wurde der Workshop vom Autor dieser Arbeit ${ }^{154}$ und einem weiteren Kollegen.

Nach einer Einleitung zu Zielen und Zeitplanung des Projektes, wurden in einer ersten Runde spontane Eindrücke, didaktische Anregungen und Kritikpunkte bezüglich der Projektziele, der Zeitplanung und der Projektverwirklichung gesammelt ("Blitzlicht"-Statements, vgl. Lipp \& Will, 2004, S. 94 96) und anschließend zusammengefasst. Anschließend wurde ein Katalog von sieben Leitfragen ${ }^{155}$ mit Hilfe einer Kartenabfrage (zur Verwendung der Kartenabfrage in Expertenworkshops vgl. Lipp \& Will, 2004, S. 75 - 86) abgearbeitet. Die Ergebnisse der Kartenabfrage wurden aufgrund der unterschiedlichen Gewichtung, die die Workshopteilnehmer der Beantwortung der Leitfragen zumaßen, in Form thematischer Cluster zusammengefasst (zur Methode des Clusterings vgl. Lipp \& Will, 2004, S. 78), die Relevanz einzelner Statements wurden von jedem Workshopteilnehmer anschließend mit Punkten bewertet (zur Methode des Punktens vgl. Lipp \& Will, 2004, S. 110 - 115).

Nachfolgend sind ausschließlich jene Aussagen zusammengefasst, die im Konsens aller Teilnehmer als relevant erachtet wurden. Statements, bei denen keine Einigkeit der Anwesenden über Relevanz und Richtigkeit der Aussage erzielt werden konnten, wurden in der nachfolgenden Zusammenfassung nicht berücksichtigt.

154 Die Moderatoren beschränkten sich im gesamten Workshop auf die die Organisation des Ablaufs, die Erklärung der verschiedenen Abfragerunde sowie auf Verständnisfragen zu einzelnen Statements bzw. Nachfragen, wenn bestimmte Punkte des Leitfadens nicht bearbeitet worden waren.

155 Leitfragen: (1) Welche didaktischen Methoden bieten sich in welcher Reihenfolge an? (2) Wie viele Unterrichtsstunden sollten aus Expertensicht mindestens für die medienpädagogische Intervention verwendet werden? (3) Wie muss das Unterrichtsmaterial gestaltet sein? (4) Welche Mehrarbeit kommt durch den medienpädagogischen Unterricht auf die Beteiligten (Lehrer, Aufsichtsbehörden, Eltern) zu? (5) Wie kann innerhalb des Unterrichtskonzeptes ein Höchstmaß an Standardisierung erreicht werden, ohne dass sich Lehrende in ihren Möglichkeiten zu stark eingeschränkt fühlen? (6) Wie müsste die Lehrerfortbildung gestaltet sein, dass sie eine bestmögliche Mischung aus Effektivität und Teilnahmemotivation bietet? (7) Wie können Eltern möglichst effektiv in den Unterricht mit eingebunden werden? 
9.2.1 Cluster 1: Implementierung des Unterrichtskonzeptes in organisatorische Strukturen

Die teilnehmenden Experten waren sich einig darüber, dass ein Unterrichtskonzept zur Prävention problematischer Mediennutzungsmuster im Kindesalter möglichst enge Bezüge zu den landesspezifischen Rahmenlehrplänen ausweisen sollte, um auch auf Seiten der Schulbehörden und der Schulleitungen akzeptiert $\mathrm{zu}$ werden. Hier wurde allgemein eine große Zustimmung zu den Projektinhalten auf Seiten von Schulbehörden und Schulleitungen erwartet, was auch für wichtig erachtet wurde, um weniger motivierte Lehrkräfte von der Teilnahme an dem Projekt zu überzeugen. Dieser Punkt sei auch deshalb von großer Bedeutung, da die Begleitforschung zum Erfolg des Konzeptes von der Genehmigung der Schulbehörden und der Schulleitungen abhängig sei. Zudem wurde angeregt, bei der erforderlichen Fortbildung der Lehrkräfte eng mit den zuständigen Fortbildungsstellen zu kooperieren, damit die Veranstaltung als offizielle LehrkräfteFortbildung anerkannt wird. Dies wurde auch als wichtig für die Motivation der Lehrkräfte bei der Teilnahme an dem Präventionskonzept erachtet.

\subsubsection{Cluster 2: Lehrkräftefortbildung und Lehrkräftemotivation}

Alle Teilnehmerinnen und Teilnehmer stimmten darin überein, dass es wichtig sei, teilnehmenden Lehrkräften ein „fertiges Unterrichtspaket“ vorzulegen, das wenig eigene Entwicklungsarbeit auf Seiten der Lehrkräfte erfordert. Dies sei auch deshalb wichtig, um mögliche Effekte des Präventionsprojektes auf das konkrete Unterrichtskonzept zurückführen zu können. Es wurde jedoch auch betont, dass Lehrkräfte mit einem fertigen Konzept nicht einfach ,überrumpelt“ werden dürften, da dies bei Unklarheiten oder Ablehnung einer Konzeptinhalte schnell zur Ablehnung des gesamten Konzeptes beitragen können. Insofern solle das Konzept im Vorhinein noch einmal mit einigen Lehrkräften besprochen und aufgrund ihres kritischen Feedbacks angepasst werden. Als durchaus hilfreich wurde es von allen Teilnehmerinnen und Teilnehmern des Workshops erachtet, den Lehrkräften das SMART-Projekt ${ }^{156}$ vorzustellen, um einem verbreiteten Skeptizismus zu begegnen, durch Schulprojekte kindliche Verhaltensmuster kaum ändern zu können. Andererseits wurde aber betont, inhaltlich solle das Konzept auf Botschaften und Methoden beruhen, die für Deutschland als erfolg- 
versprechend angesehen würden und insofern teilweise andere Wege als das SMART-Projekt gehen.

\subsubsection{Cluster 3: Didaktik, Methoden und Materialien}

Ausdrücklich unterstrichen wurde von den Teilnehmerinnen und Teilnehmern des Workshops, dass das Thema des Unterrichts, die Nutzung von Medien, keineswegs zu einem starken Einsatz von elektronischen Medien im Unterricht führen müsse. Vielmehr müsse der Grundsatz gelten, dass die Verwendung der Medien immer von den Inhalten und didaktischen Zielen bestimmt sei. Der gezielte Einsatz neuer Medien könne allerdings zuweilen einen wichtigen Impuls setzen, um das Interesse der Schülerinnen und Schüler zu wecken. Es müsste aber immer bedacht werden, dass der Einsatz Neuer Medien, zumal interaktiver Medien, spezielle Ressourcen auf Seiten der Schule voraussetzt, die nicht unbedingt überall gegeben seien und darüber hinaus zeitaufwändig sein könne. Dieser Zeitaufwand dürfe nicht zulasten wichtiger didaktischer Ziele gehen. Sehr begrüßt wurde die Idee der Verwendung eines Medientagebuches im Rahmen des Unterrichts, um eine Sensibilisierung der Schülerinnen und Schüler für die eigene Mediennutzung zu erreichen und Grundlage einer Diskussion in der Klasse über die Mediennutzung der Kinder zu schaffen. Außerdem könne mit einem Medientagebuch ein Impuls dahingehend gesetzt werden, dass die Schülerinnen und Schüler selbst kleine Forschungsprojekte zur Mediennutzung in ihrem Umfeld durchführen und die Ergebnisse in der Klasse vorstellen. Allgemein befürwortet wurde die Verwendung von Klassenvereinbarungen beziehungsweise Verträgen zur Mediennutzung nach dem SMART-Konzept, solange diese Vereinbarungen flexibel gestaltbar seien und somit Besonderheiten in den Klassen berücksichtigt werden könnten.

\subsubsection{Cluster 4: Zeitliche Planung und Gestaltung des Unterrichtes}

Die Teilnehmerinnen und Teilnehmer unterstützten zwar die Meinung, das Unterrichtsprojekt dürfe keinen unzumutbar breiten Raum im Unterricht einnehmen, so dass die Vermittlung anderer Unterrichtsstoffe zu kurz kommen würde, andererseits sei eine zu große zeitliche Begrenzung nicht sinnvoll (die in einem Statement aufgeworfenen Frage, ob eine erste Unterrichtseinheit mit einem Umfang von acht Schulstunden ausreichend sei, wurde allgemein verneint). Als idealer zeitlicher Rahmen für eine Lehrkräftefortbildung zur Durch- 
führung der Unterrichtseinheit wurde ein zeitlicher Rahmen von drei, maximal vier Stunden erachtet. Wichtig sei es aber, die Unterrichtseinheit zu einer Zeit durchzuführen, in der der „Prüfungs- und Notendruck“ weniger groß sei. Zudem sei in diesen Zeiten (um die Weihnachtszeit sowie einige Wochen vor den Sommerferien) auch der elterliche Terminplan sehr voll, so dass eine effiziente Einbindung der Eltern in den Medienunterricht sich schwierig gestalten könne.

\subsubsection{Cluster 5: Einbindung der Eltern}

Bei der Frage, in welchem Rahmen Eltern in die Gestaltung der Unterrichtsintervention einbezogen werden könnten, wurde die Verwendung von Elterninformationsbroschüren und Elternbriefen zwar nicht abgelehnt, dennoch sei die Veranstaltung von Elternabenden das wichtigste Instrument zur Einbeziehung von Eltern. Dieser Elternabend müsse zeitlich kurz vor oder kurz nach Beginn der Unterrichtseinheit platziert werden. Zwar gebe es bei Elternabenden immer wieder das Problem, dass insbesondere Eltern problematischer Schüler nicht teilnehmen würden (wobei dieses Problem in der Grundschule nicht so stark ausgeprägt sei, wie in der Sekundarstufe), dies könne aber unter Umständen dadurch vermieden werden, keinen Themenelternabend zur Medienerziehung anzubieten, sondern die entsprechenden Informationen bei einem „regulären“ Elternabend $\mathrm{zu}$ geben, der wegen der $\mathrm{zu}$ treffenden organisatorischen und finanziellen Entscheidungen zumeist besser besucht sei. Eine hohe Elternbeteiligung sei auch insofern wichtig, da für eine Teilnahme der Schülerinnen und Schüler an der Begleitforschung im Grundschulalter die ausdrückliche elterliche Zustimmung eingeholt werden müsse und ein Elternabend ein gutes Forum sei, Eltern von der Wichtigkeit der Untersuchung zu überzeugen. Außerdem würde dies wahrscheinlich auch die Elternbeteiligung an der Begleitforschung (Elternfragebögen) erhöhen. Zudem wurde von allen am Workshop Beteiligten dafür plädiert, den Elternabend unter Mitwirkung eines externen Experten zur kindlichen Mediennutzung durchzuführen, da die Anwesenheit einer solchen Person erfahrungsgemäß die Teilnahmemotivation an der Veranstaltung und die Aufmerksamkeit während der Veranstaltung erhöhe.

\subsection{Konzeption und Evaluation des Medienlotsen-Unterrichtsprogramms}

In den nachfolgenden Unterkapiteln wird die Konzeption von drei Unterrichtseinheiten für Schülerinnen und Schüler der dritten, vierten und fünften Klasse 
dargestellt. Die drei Unterrichtseinheiten des Medienlotsen-Programms tragen die Namen Vom Leichtmatrosen vom Medienlotsen, Die ELIZA-Protokolle sowie Medienlotsen gesucht! Die Unterrichtseinheiten bauen, obwohl inhaltlich weitgehend voneinander unabhängig, auf die Ergebnisse der jeweils vorangegangenen Unterrichtseinheit auf. Die Entwicklung und Implementierung der Unterrichtseinheiten wurde von Anfang an als „lernendes Modell“ konzipiert, so dass das Feedback der Lehrkräfte im Rahmen der Konzeption und Organisation der Unterrichtseinheiten an möglichst vielen Abschnitten berücksichtigt werden konnte. In Tabelle 4 auf S. 245 sind die einzelnen Konzeptions- und Evaluationsschritte für alle drei Unterrichtseinheiten dargestellt.

Jeder Unterrichtseinheit ging ein Workshop mit den beteiligten Lehrkräften voraus, in dem erste Ergebnisse der Schülerbefragungen zum letzten Messzeitpunkt und auch erste Erkenntnisse zu Effekten der vorangehenden Unterrichtseinheiten berichtet wurden. Darauf aufbauend wurde gemeinsam mit den Lehrkräften das Konzept der neuen Unterrichtseinheit entwickelt. Dabei wurden vom Autor dieser Arbeit die medienerzieherischen Ziele der Unterrichtseinheit präsentiert und ein konzeptioneller Vorschlag mit Umsetzungsbeispielen in den Workshop eingebracht. Konzeptioneller Vorschlag sowie Umsetzungsvorschläge wurden im Anschluss vor dem Hintergrund didaktischer, inhaltlicher und organisatorischer Aspekte diskutiert. Auf Grundlage der Workshopprotokolle wurden dann alle Unterrichtsmaterialien und Begleittexte vom Autor dieser Arbeit fertig gestellt und den Lehrkräften in fertigen Klassensätzen zur Verfügung gestellt. 
Tabelle 4: Überblick über die wichtigsten Konzeptions- und Evaluationsschritte des Medienlotsen-Programms

\begin{tabular}{|c|c|c|c|}
\hline $\begin{array}{l}\text { Name der Unter- } \\
\text { richtseinheit }\end{array}$ & $\begin{array}{l}\text { Vom Leicht- } \\
\text { matrosen zum } \\
\text { Medienlotsen } \\
\end{array}$ & $\begin{array}{l}\text { Die ELIZA- } \\
\text { Protokolle }\end{array}$ & $\begin{array}{l}\text { Medienlotsen ge- } \\
\text { sucht! }\end{array}$ \\
\hline Klasse & 3. Klasse & 4. Klasse & 5. Klasse \\
\hline $\begin{array}{l}\text { Vorbereitungs- } \\
\text { workshop }\end{array}$ & Februar 2006 & Februar 2007 & Februar 2008 \\
\hline $\begin{array}{l}\text { Durchführung der } \\
\text { Unterrichtseinheit }\end{array}$ & März bis Mai 2006 & März bis Mai 2007 & März bis Mai 2008 \\
\hline Pretest & $\begin{array}{l}\text { November } 2005 \text { bis } \\
\text { Februar } 2006 \text { an } 44 \\
\text { Klassen in Olden- } \\
\text { burg und Soltau- } \\
\text { Fallingbostel }\end{array}$ & -- & -- \\
\hline \multirow[t]{3}{*}{ Evaluationsschritte } & $\begin{array}{l}\text { Konzeptevaluation } \\
\text { im Rahmen des } \\
\text { Pretests im } \\
\text { November } 2005\end{array}$ & -- & -- \\
\hline & $\begin{array}{l}\text { Formative } \\
\text { Evaluation im An- } \\
\text { schluss an den Pre- } \\
\text { test im Februar } \\
2006\end{array}$ & -- & -- \\
\hline & $\begin{array}{l}\text { Konzeptevaluation } \\
\text { im Rahmen der } \\
\text { Vorbereitungs- } \\
\text { workshops im } \\
\text { Februar } 2006\end{array}$ & $\begin{array}{l}\text { Konzeptevaluation } \\
\text { im Rahmen der } \\
\text { Vorbereitungs- } \\
\text { workshops im } \\
\text { Februar } 2007\end{array}$ & $\begin{array}{l}\text { Konzeptevaluation } \\
\text { im Rahmen der } \\
\text { Vorbereitungs- } \\
\text { workshops im } \\
\text { Februar } 2008\end{array}$ \\
\hline
\end{tabular}


Tabelle 4 (fortgesetzt)

\begin{tabular}{|c|c|c|c|}
\hline $\begin{array}{l}\text { Name der } \\
\text { Unterrichts- } \\
\text { einheit }\end{array}$ & $\begin{array}{l}\text { Vom Leicht- } \\
\text { matrosen zum } \\
\text { Medienlotsen }\end{array}$ & $\begin{array}{l}\text { Die ELIZA- } \\
\text { Protokolle }\end{array}$ & $\begin{array}{l}\text { Medienlotsen ge- } \\
\text { sucht! }\end{array}$ \\
\hline \multirow{11}{*}{$\begin{array}{l}\text { Evaluations- } \\
\text { schritte }\end{array}$} & Evaluation der & Evaluation der & Evaluation der \\
\hline & Programmdurch- & Programmdurch- & Programmdurch- \\
\hline & führung auf Grund- & führung auf Grund- & führung auf Grund- \\
\hline & lage einer Lehr- & lage einer Lehr- & lage einer Lehr- \\
\hline & kräftebefragung im & kräftebefragung im & kräftebefragung im \\
\hline & Mai/Juni 2006 & Mai/Juni 2007 & Mai/Juni 2008 \\
\hline & Ergebnisevaluation & Ergebnisevaluation & Ergebnisevaluation \\
\hline & im Rahmen der & im Rahmen der & im Rahmen der \\
\hline & summativen & summativen & summativen \\
\hline & Evaluation des & Evaluation des & Evaluation des \\
\hline & Gesamtkonzeptes & Gesamtkonzeptes & Gesamtkonzeptes \\
\hline
\end{tabular}

Da mit der Entwicklung und Umsetzung des MedienlotsenMedienunterrichtskonzeptes in vielen Bereichen Neuland beschritten wurde, erfolgte im Anschluss an die Konzeption der ersten Unterrichtseinheit eine Erprobung des Programms in 40 norddeutschen Schulklassen (in der Stadt Oldenburg und im Landkreis Soltau-Fallingbostel), die von einer formativen Evaluation begleitet wurde (zu den Ergebnissen dieser Evaluation vgl. die Ausführungen ab S. 259 in dieser Arbeit). Aus organisatorischen Gründen wurden die Unterrichtseinheiten beim Konzepttest in Norddeutschland in vierten Klassen erprobt, deren Schülerschaft zum Zeitpunkt der Programmdurchführung (November 2005 bis Februar 2006) etwas älter war (im Durchschnitt 6 Monate älter), als die Schülerschaft der dritten Klassen im Berliner Längsschnitt Medien bei der Programmdurchführung (März bis Mai 2006).

Erst im Anschluss an die Erprobung wurde das Unterrichtskonzept im Rahmen des Berliner Längsschnitt Medien eingesetzt. Im Anschluss an die Durchführung jeder Unterrichtseinheit im Rahmen des Berliner Längsschnitt Medien wurde eine Evaluation der Programmdurchführung mithilfe einer Lehrkräftebefragung durchgeführt. Die Entscheidung, die Programmdurchführung mithilfe von Daten einer Lehrkräftebefragung zu evaluieren, erfolgte einerseits aus forschungsökonomischen, andererseits auch aus sachlichen und methodischen Gründen. So wäre es theoretisch möglich gewesen, auch die Schülerinnen und Schüler selbst im Rahmen der auf die drei Unterrichtseinheiten folgenden Messzeitpunkte (MZP2, MZP3, MZP4) zu ihren Meinungen über das 
Unterrichtskonzept oder ihrem medien- und medienwirkungsbezogenen Wissen zu befragen. Einerseits hätte eine solche Befragung den ohnehin schon beträchtlichen Untersuchungsaufwand vergrößert, was langfristig gesehen neben forschungsökonomischen Gesichtspunkten auch zu höherer Untersuchungsmüdigkeit geführt hätte. Zudem wurde auf Seite 229 in dieser Arbeit dargestellt, dass als maßgebliches Kriterium der Implementationsfähigkeit eines Unterrichtsprogramms zur Prävention problematischer Mediennutzungsmuster im Kindesalter die Bereitschaft der Lehrkräfte angesehen wird, ein solches Unterrichtsprogramm durchzuführen. 


\subsubsection{Das erste Unterrichtskonzept (3. und 4. Klassen)}

In Kapitel 8 (S. 207 - 210) wurde bereits deutlich, dass ein Präventionskonzept inhaltlich und didaktisch so gestaltet werden sollte, dass es sowohl im Deutsch/Sprachunterricht als auch im Sachunterricht eingesetzt werden kann, idealerweise in beiden Fächern. Das erfolgreiche Beispiel des amerikanischen SMARTKonzeptes (vgl. S. 211 - 226 in dieser Arbeit) hat gezeigt, dass die Vermittlung positiver Rollenvorbilder, deren Verstehen und angeleiteter Nachvollzug geeignete Mittel zur Verhaltensänderung von Schülerinnen beziehungsweise Schülern sein können, wenn entsprechende motivationale Verstärker eingesetzt werden (zum Beispiel durch die Belohnung wichtiger Teilerfolge oder die Stärkung des Zusammengehörigkeitsgefühls in der Klasse). Es wurde bereits zuvor dargestellt, dass Modell-Lernen immer dann besonders erfolgreich ist, wenn die Verhaltensmodelle in alltagsnahen Settings platziert sind, die der Lebenswelt der Schülerinnen und Schüler weitgehend entsprechen. Im Rahmen der Auseinandersetzung mit dem SMART-Konzept wurde aber auch kritisch angemerkt, dass die Prävention inhaltlich problematischer Mediennutzungsmuster im Rahmen des Programms nicht ausreichend berücksichtigt wurde und dass die Rolle der Lehrkraft im SMART-Programm in dieser Form nur schwerlich auf Deutschland übertragbar sein wird (vgl. auch die ab S. 226 formulierte Kritik am SMART-Programm). Es wurde darüber hinaus gezeigt, dass gerade Grundschullehrkräfte in Deutschland nur wenig konkretes Wissen über die alltägliche Mediennutzung ihrer Schüler haben und ihre durchaus medienkritische Haltung sehr unspezifisch ist, was sie deutlich vom Idealbild eines „Engagierten Profis“ in der Medienerziehung unterscheidet (vgl. S. 169 - 173 in dieser Arbeit). Andererseits wurde von den Beteiligten des Expertenworkshops darauf hingewiesen, dass eine Fortbildung der Lehrkräfte im Vorfeld der Unterrichtsprävention nur einige Stunden dauern sollte und dass auf dieser Grundlage bereits die Durchführung der Unterrichtsprävention möglich sein sollte (vgl. S. 242 in dieser Arbeit).

\subsubsection{Unterrichtsmaterial}

Vor dem Hintergrund der oben beschriebenen Anforderungen und Herausforderungen wurde das Unterrichtskonzept Vom Leichtmatrosen zum Medienlotsen entwickelt. Als zentrales Unterrichtsmedium wurde die Form einer Lesegeschichte für Kinder gewählt, die sowohl im Sachunterricht als auch im Deutschunterricht einsetzbar ist. Dies geschah insbesondere aus dem Grund, da 
die Arbeit mit Texten Lehrkräften bestens vertraut ist und somit das vielen Lehrkräfte mutmaßlich wenig vertraute Thema der kindlichen Alltagsmediennutzung mit einem vertrauten didaktischen Medium verknüpft wird. Zudem wurde auch im Expertenworkshop darauf hingewiesen, dass der Einsatz Neuer Medien, zumal interaktiver Medien, spezielle Ressourcen auf Seiten der Schule voraussetzt, die nicht unbedingt überall gegeben sind und darüber hinaus zeitaufwändig sein kann (vgl. S. 242 in dieser Arbeit). Während der Einsatz spezieller Kinder- und Jugendliteratur im Deutschunterricht breit etabliert und didaktisch ausgearbeitet ist (vgl. Jesch, 2005) hat sich auch in der Sachunterrichtsdidaktik die Meinung durchgesetzt, dass neben kindgerechten Sachtexten und -büchern auch fiktionale Texte ,zur Erschließung von Lebenswirklichkeit“ beitragen können (Gläser, 2007, S. 164), wobei der Inhalt ,an der Alltagswelt und den Erfahrungen der Kinder anknüpfen [sollte] und darüber hinaus möglichst Identifikationsmöglichkeiten bieten [sollte]“ (Ehlers, 2004, S. 119). Dabei sollten nach Ehlers Handlungsmöglichkeiten eröffnet werden, „so dass mit dem Buch im wahrsten Sinne des Wortes umgegangen werden kann.“(ebenda). Wedel-Wolff betont dabei die möglichst individuelle Ausrichtung von Texten: „Das, was das Kind selbst betrifft, was es persönlich angeht, das ist wichtig und interessant.“ (2006, S. 19). Ehlers hebt bei der Verwendung von fiktionalen Texten im Sachunterricht besonders die Rolle des Vorlesens der Texte hervor:

„Leider und zu Unrecht ist das Vorlesen lange Zeit sozusagen ,aus der Mode gekommen', sowohl in der Familie, aber auch in der Schule. Hier wie da fehlt angeblich die Zeit. Die positiven Effekte des Vorlesens werden nicht genügend gewürdigt. [Auch] in den meisten Lehrplänen wird das Vorlesen eher wenig beachtet. Erst in jüngster Zeit hat die bereits genannte PISA-Studie das Vorlesen wieder weiter in das Zentrum des Interesses gerückt. [...] Das Vorlesen sollte in den Schulen wieder einen grundsätzlich größeren Stellenwert erhalten und sich nicht nur auf das Vorlesen während der Frühstückspause beschränken. [...] Auf gar keinen Fall darf das Vorlesen aber für Kinder zu einem langweiligen oder gar beängstigenden Ereignis werden. Wir alle erinnern uns vermutlich noch an die peinlichen Momente in der Schule, wenn ein vorlesendes Kind ins Stocken geriet oder sich mühsam von Zeile zu Zeile vorarbeitete. [...] Es erscheint sinnvoll, Lesen und den Umgang mit Büchern nicht als reine Technik zu üben, sondern das Lesen sollte immer mit ansprechenden Inhalten zu tun haben und einen Lustgewinn bedeuten.“ (Ehlers, 2004, S 117)

Reinhard (2003, S. 18 - 22) betont im Hinblick auf die Verwendung von Geschichten in pädagogischen Kontexten, dass Geschichten den Rezipienten wichtige Sekundärerfahrungen vermitteln können, wenn Primärerfahrungen nicht möglich sind. Im Rahmen präventiver Maßnahmen, in denen es regelmäßig um die Vermeidung bestimmter Handlungs- oder Einstellungsmuster geht, sind Primärerfahrungen mit dem Unterrichtsgegenstand indes häufig nicht nur nicht möglich, sondern sogar ausdrücklich nicht erwünscht. Insofern eignen sich Lesegeschichten aufgrund ihrer Möglichkeit der Thematisierung problematischer 
Inhalte ganz besonders, da durch die rein textliche Vermittlung der Inhalte einerseits eine hinreichend plastische Darstellung des Geschehens und andererseits eine hinreichend distanzierte Form der Rezeption ermöglicht wird.

In Ermangelung geeigneter Kinderliteratur oder geeigneter Lehrbuchtexte für Kinder, die sich mit dem Thema der kindlichen Mediennutzung auseinandersetzen, wurde vom Autor dieser Arbeit eine Lesegeschichte konzipiert und geschrieben. Die sprachliche und inhaltliche Gestaltung der Geschichte sollte einen Einsatz sowohl in der dritten als auch in der vierten Grundschulklasse ermöglichen. Im Rahmen der Geschichte wird der Lernprozess einer fiktionalen Schulklasse und ihrer Klassenlehrerin zum Thema „Mediennutzung von Kindern“ nachvollzogen. Die Lesegeschichte rankt sich dabei um drei zentrale Instrumente der Medienerziehung: ein Medientagebuch, einen Mediennutzungsplan und einen Medienvertrag, die auch im Rahmen des SMART-Projektes genutzt wurden (vgl. S. 211 - 226). In 15 Mini-Episoden wird die Geschichte der Klasse 4b erzählt, die mit ihrer Klassenlehrerin Frau Siebrecht eine Klassenfahrt an die Nordsee macht (vgl. auch die Textbeispiele in Tabelle 5, S. 252). In einer im Prinzip „medienfreien“ Umgebung brechen zwischen den Schulkindern und ihrer Lehrerin während des Aufenthaltes in der Jugendherberge immer wieder Konflikte über die Medien der Kinder auf. Sie beschweren sich, dass sie bestimmte Fernsehsendungen verpassen, bestimmte Videospiele nicht spielen können. Dabei zeigt sich schnell, dass es zwischen Schülern und Lehrerin große Unterschiede in der Nutzung und Bewertung von Medien gibt. Während sich die Lehrerin Frau Siebrecht Sorgen macht, dass ihre Klasse zu viel vor dem Fernseher oder dem Computer sitzt, verteidigt Leon, einer ihrer Schüler, immer wieder seine Freude am stundenlangen Fernsehen und Computer spielen. Außerdem zeigt sich, dass auch Erwachsene, Eltern und Lehrer, auf Medien wie das Fernsehen nicht verzichten wollen und häufig keine guten Vorbilder für die von ihnen kritisierten Kinder sind. Frau Siebrecht stellt den Kindern die Methode das Medientagebuches vor und lässt die Kinder auf Grundlage eines retrospektiven Tagebuches der letzten Woche vor der Klassenfahrt erheben, wie lange sie täglich elektronische Medien nutzen. Die Leuchtturmwärterin Anneliese, die von der Klasse im Rahmen eines Ausflugs besucht wird, vermittelt schließlich zwischen den Kindern und ihrer Lehrerin. Sie bringt Frau Siebrecht auch auf die Idee, wie man die Kinder zu einer bewussten Mediennutzung bewegen kann, ohne die Medien zu verteufeln. Dabei wird die Notwendigkeit betont, Medienfreizeit und nicht-mediale Freizeitgestaltung in ein ausgewogenes Verhältnis zu bringen. Anneliese stellt am Beispiel des Fernsehplans eine Methode vor, die Mediennutzung langfristig zu planen und zu begrenzen (vgl. Tabelle 5, Textbeispiel 5). Wieder in der Schule zurück, schließt die Klasse 4b einen Medienver- 
trag, in dem die Kinder zusammen mit der Lehrerin festlegen, wie sie in Zukunft mit dem Fernseher und dem Computer umgehen sollen. Der genaue Inhalt des Vertrages bleibt genauso offen wie auch viele andere Details.

Für jede Lese-Episode wird den Lehrkräften eine Reihe von Verständnisfragen für die Schülerinnen und Schüler mitgegeben. Zudem werden Vorschläge für Aktivitäten und Diskussionen in der Klasse gemacht. Dabei vollziehen die Klassen, in denen das Unterrichtskonzept durchgeführt wird, Schritt für Schritt die Aktivitäten und Diskussion der Klasse 4b in der Lesegeschichte nach. Auch die Schülerinnen und Schüler in der Unterrichtsklasse führen ein Medientagebuch, entwickeln Mediennutzungspläne und schließen am Ende der Unterrichtseinheit einen Medienvertrag. Vor dem Hintergrund der vermuteten defizitären Kenntnisse vieler Lehrkräfte über alltägliche kindliche Mediennutzungsmuster wurde im Rahmen der Geschichte ein gegenseitiger Lernprozess inszeniert, in dessen Rahmen die Lehrerin zunächst von den Kindern lernt, welche Medien für sie von Bedeutung sind und in welchem Ausmaß diese Medien genutzt werden (vgl. Tabelle 5, Textbeispiele 1, 2 und 5). Die Kinder wiederum lernen durch ihre Lehrerin Verfahren kennen, mit deren Hilfe sie erkennen, wie stark ihr Alltag von Mediennutzung geprägt ist (vgl. Tabelle 5, Textbeispiel 5). Zudem wird ihre Mediennutzung von der Lehrerin mitunter kritisch kommentiert, wobei die Kommentare zunächst sehr unspezifisch-kritisch sind, dann aber - mit zunehmendem Lernprozess der Lehrerin - immer treffender werden. Darüber hinaus bringt der Dialog mit der Lehrerin auch Gespräche unter den Kindern in Gang. Wenn sie auch vor ihrer Lehrerin nicht gerne zugeben, dass deren Kritik in einigen Punkten stimmen könnte, erzählen sie sich untereinander durchaus von unangenehmen Medienerfahrungen (vgl. Tabelle 5, Textbeispiele 3 und 4). 
Tabelle 5: Textbeispiele aus der Lesegeschichte Vom Leichtmatrosen zum Medienlotsen

\section{Textbeispiel 1: Kinder erklären ihre Medien}

Leon fühlt sich sofort angesprochen. „Ich habe überhaupt keinen Computer!“ ruft er. „Aber eine Playstation“, ruft Aische. „Das ist doch das Gleiche.“ „Du hast doch überhaupt keine Ahnung“, sagt Leon. „Eine Playstation ist eine Spielkonsole und ein Computer ist... eben ein Computer." „Was ist denn der Unterschied?“ erkundigt sich Frau Siebrecht. Sie weiß es nämlich auch nicht genau. „Eine Spielkonsole ist nur zum Spielen da“, sagt Markus noch bevor Leon antworten kann. „Mit einem Computer kann man alles Mögliche machen. Im Internet surfen, Texte schreiben, Fotos angucken, alles eben.“ „Dann ist ein Computer doch viel besser, oder?" fragt Frau Siebrecht. „Nö, der ist nämlich viel komplizierter und es dauert viel länger, bis man ihn gestartet hat. Mit meiner Spielkonsole könnte sogar meine Mutter umgehen", antwortet Leon und die Klasse lacht.

\section{Textbeispiel 2: Erfolg beim Computerspiel}

Frau Siebrecht hat eine neue Aufgabe für die Kinder. Sie möchte, dass jeder aufschreibt, was an Computerspielen gut ist und was nicht so gut ist. Damit es leichter für die Kinder wird, bittet Frau Siebrecht sie, ein schönes Erlebnis mit einem Computerspiel aufzuschreiben und ein nicht so schönes Erlebnis. Zehn Minuten lang ist es mucksmäuschen-still im Raum, weil alle Kinder angestrengt nachdenken und schreiben. Dann dürfen die Kinder der Reihe nach vorlesen, was sie aufgeschrieben haben. Leon hat Folgendes geschrieben: „Schön war, als ich zum ersten Mal gegen meinen Bruder Need for Speed gewonnen habe. Das ist ein Autorennspiel", fügt er hinzu, als ihn seine Lehrerin fragend anguckt. Er liest weiter. „Ich habe ganz lange geübt, weil mein Bruder fast ein Jahr älter ist als ich und vorher immer gewonnen hat. Aber dann habe ich gewonnen und seitdem gewinne ich fast immer." 


\section{Textbeispiel 3: Isolation}

„Ich habe einen Onkel Alex“, wispert Daniel, „der ist bloß zehn Jahre älter als ich. Das kommt daher, weil er der jüngste Bruder meiner Mutter ist. Und Alex spielt die ganze Zeit solche Spiele, von denen Sarah eben erzählt hat.“ „Cool!“ sagt Leon fast schon laut. „Nee, überhaupt nicht cool“, flüstert Daniel zurück. „Meine Mutter hat zu meinem Vater gesagt, dass sie sich ganz große Sorgen um Alex macht, weil er fast nur noch in seinem Zimmer sitzt und kaum mehr mit anderen Leuten spricht.“ [...] Leon ist für eine Weile still. [...] Schließlich dreht er sich zu Daniel um. „Glaubt du denn wirklich, dass dein Onkel vom Computer spielen so komisch geworden ist?" fragt er. „Keine Ahnung“, sagt Daniel. „Ich glaube, Mama hat mal gesagt, dass Alex schon als Kind Schwierigkeiten hatte, Freunde zu finden. Aber durch das Computer spielen hat er sich bestimmt noch weniger bemüht als vorher.“ „Zum Glück hab ich ja schon Freunde", meint Leon schließlich und gähnt herzhaft. „Bevor ich so werde wie dein Onkel Alex, müsst ihr mich einfach warnen.“ „Machen wir“, flüstert Markus zurück, bevor er einschläft. „Kannste dich drauf verlassen.“

\section{Textbeispiel 4: Angst}

Frau Siebrecht sieht, dass Sarah ganz klein auf ihrem Stuhl geworden ist. „Na, Sarah, möchtest du nicht vorlesen?“ „Ich habe aber kein schönes Erlebnis aufgeschrieben, sondern nur ein schlimmes“, sagt Sarah leise. Dann liest sie vor: „Einmal hat mein Bruder mir ein Computerspiel gezeigt, das ganz schlimm war. Da waren überall böse Monster, die man abschießen musste und man hat richtig ihr Blut gesehen. Und die ganze Zeit war unheimliche Musik. [...] Danach habe ich die ganze Nacht schlecht geträumt und durfte schließlich bei meinen Eltern im Bett schlafen. „Memme!“ zischt Daniel ihr zu, aber Frau Siebrecht hat es gehört. „Daniel, du bist ganz schön gemein“, tadelt sie ihn. „Ich möchte, dass ihr heute Nachmittag alle ein Bild malt. Es soll ein Bild sein, über etwas, was ihr im Fernsehen oder beim Computer spielen gesehen habt und was euch richtig Angst gemacht hat." 


\section{Textbeispiel 5: Die Eltern und der Fernsehplan}

Am Ende der nächsten Woche reden die Kinder der $4 \mathrm{~b}$ am Freitagmittag noch einmal mit Frau Siebrecht über das Thema Medien. Alle berichten, ob sie es geschafft haben, ihre Fernsehliste einzuhalten. Einige haben die Liste nicht ganz genau eingehalten, aber dafür an einem anderen Tag eine Sendung gestrichen. Selbst Leon hat an zwei Tagen gar kein Fernsehen geguckt. „Ich hab mich aber ganz schön heftig mit meinen Eltern rumgestritten“, sagt er. „Mama lässt den Fernseher nämlich immer in der Küche laufen, wenn sie was kocht. Und als sie wollte, dass ich helfe, hab ich ihr gesagt, dass die Sendung, die gerade im Küchenfernseher lief, nicht auf meinem Plan steht. Da hat sie ihn schließlich ausgemacht. Aber als Papa und Mama einmal abends eine Serie beim Essen gucken wollten, haben sie den Fernseher nicht abgeschaltet. Ich bin ganz schön ausgeflippt, weil das doch mein fernsehfreier Tag werden sollte. Aber Pustekuchen. Ist aber echt nicht meine Schuld!“

\section{Textbeispiel 6: Alternativen}

„Sehen Sie, Frau Siebrecht, ein Computer ist nämlich manchmal auch sehr hilfreich!“ ruft Leon. „Man kann zum Beispiel etwas erst am Computer üben und es dann im normalen Leben anwenden. Mein Bruder lernt schon Englisch, und der hat ein Computerprogramm, mit dem man Englisch üben kann." Da muss Frau Siebrecht ihm Recht geben. Die Klasse überlegt eine Zeit lang, was man mithilfe von Medien noch Nützliches machen kann. Nur Sarah ist immer noch schlecht gelaunt. Damit sie wieder bessere Laune bekommt, lässt Frau Siebrecht sie und ein paar andere Kinder die lustigsten Stellen aus ihrem persönlichen Logbuch der letzten Woche vorlesen. Und schließlich stellt Daniel seine Lieblingsfreizeitbeschäftigung vor: Handball spielen. Als er davon erzählt, können alle sehen, wie seine Augen zu leuchten beginnen. Weil die erste Stunde bald schon vorbei ist und Frau Siebrecht auch noch normalen Unterricht machen will, verspricht sie den Kindern, dass die anderen ihre Lieblingsbeschäftigungen im Laufe dieser oder der nächsten Woche vorstellen dürfen, immer am Anfang einer Stunde. 
Die Konstruktion der Geschichte ist an dem Dreischritt „Medienumgang bewusst machen“ -> „Folgen des Medienumgangs erkennen“ -> „Alternativen entwickeln“ orientiert (vgl. Abbildung 4).

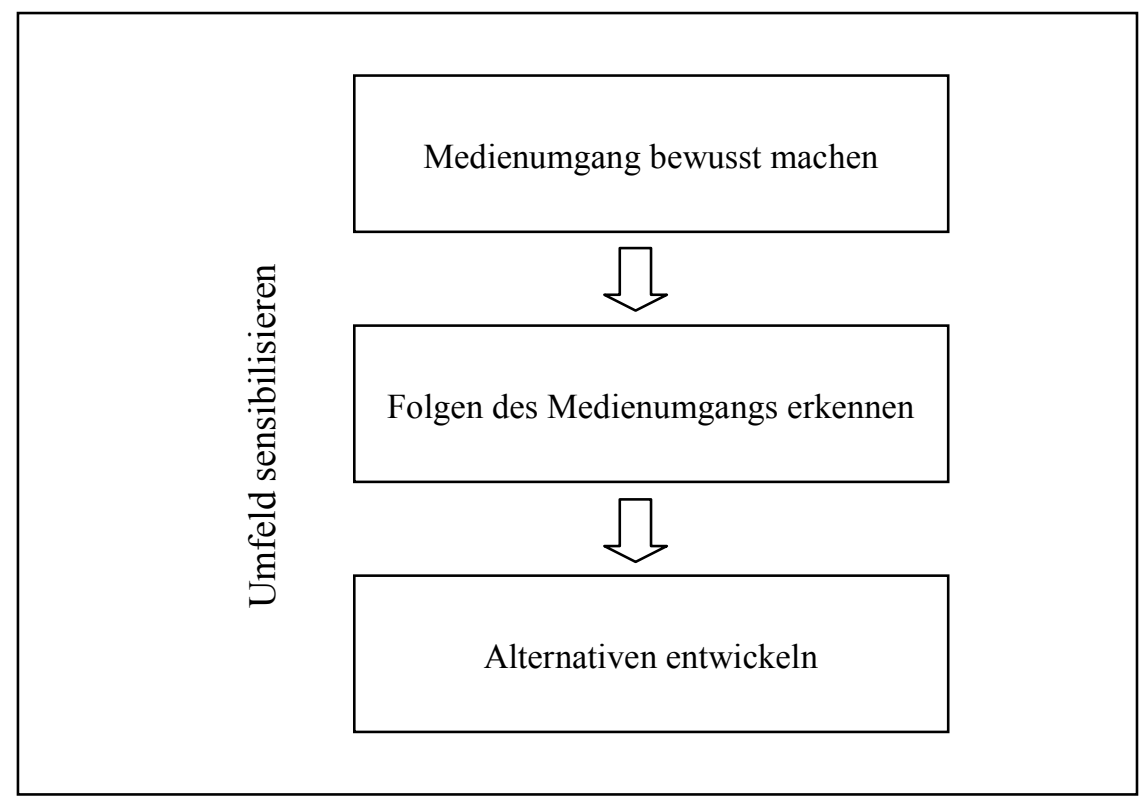

Abbildung 4: Lernschritte der Unterrichtseinheit

Da im Rahmen der Geschichte immer wieder klar wird, dass das Gelernte nur mit Unterstützung der Eltern umgesetzt werden kann, wird deutlich, dass die „Sensibilisierung des Umfeldes“ ein zentraler Stützpfeiler für den Erfolg des Medienerziehungsunterrichts ist. Vor diesem Hintergrund wird im Unterrichtsleitfaden für die Lehrkräfte die Wichtigkeit eines Elternabends zum Thema der Medienerziehung betont. In Tabelle 6 auf Seite 256 wird die episodische Struktur der Lesegeschichte Vom Leichtmatrosen zum Medienlotsen dargestellt, wobei für jedes Kapitel die angesprochenen Themen und die Stufe des Lernprozesses dargestellt werden. 
Tabelle 6: Aufbau, Themen und Lernstufen der Unterrichtseinheit Vom Leichtmatrosen zum Medienlotsen

\begin{tabular}{|c|c|c|}
\hline Kapitel & Angesprochene Themen & Dimension \\
\hline $\begin{array}{l}\text { Eine Woche ohne } \\
\text { Spongebob }\end{array}$ & - Einführung des Medienbegriffes & $\begin{array}{l}\text { Medienumgang bewusst } \\
\text { machen }\end{array}$ \\
\hline $\begin{array}{l}\text { Alles Medien oder } \\
\text { was? }\end{array}$ & $\begin{array}{l}\text { - } \text { Kategorisierung verschiedener } \\
\text { Medienarten } \\
\text { - } \quad \text { Vorstellung verschiedener } \\
\text { Mediennutzungsmodi } \\
\end{array}$ & $\begin{array}{l}\text { Medienumgang bewusst } \\
\text { machen }\end{array}$ \\
\hline $\begin{array}{l}\text { Käpt'n Fischers } \\
\text { Logbuch }\end{array}$ & - Vorstellung der Tagebuchmethode & $\begin{array}{l}\text { Medienumgang bewusst } \\
\text { machen }\end{array}$ \\
\hline Aus der Übung & $\begin{array}{l}\text { - Gründe für die Nutzung und } \\
\text { Faszination von Medien }\end{array}$ & $\begin{array}{l}\text { Medienumgang bewusst } \\
\text { machen }\end{array}$ \\
\hline $\begin{array}{l}\text { Das Freizeitlog- } \\
\text { buch }\end{array}$ & $\begin{array}{l}\text { Aufgabenbeschreibung Freizeit- und } \\
\text { Medientagebuch }\end{array}$ & $\begin{array}{l}\text { Medienumgang bewusst } \\
\text { machen }\end{array}$ \\
\hline $\begin{array}{l}\text { Wie man hundert } \\
\text { Millionen Würmer } \\
\text { zählt }\end{array}$ & $\begin{array}{l}\text { - Erklärung der wissenschaftlich- } \\
\text { empirischen Methodik } \\
\text { - Wichtigkeit genauer Messungen } \\
\text { - }\end{array}$ & $\begin{array}{l}\text { Medienumgang bewusst } \\
\text { machen }\end{array}$ \\
\hline $\begin{array}{l}\text { Wie man richtig } \\
\text { auswertet }\end{array}$ & $\begin{array}{l}\text { - Einführung in die systematische } \\
\text { Auswertung der Tagebuchangaben } \\
\text { - Vergleich eigenen Medienverhaltens } \\
\text { mit dem Medienverhalten anderer } \\
\text { Kinder }\end{array}$ & $\begin{array}{l}\text { Medienumgang bewusst } \\
\text { machen }\end{array}$ \\
\hline $\begin{array}{l}\text { Den ganzen Tag } \\
\text { vor der Spiel- } \\
\text { konsole }\end{array}$ & $\begin{array}{l}\text { - Unterscheidung Spielkonsole, } \\
\text { Computer } \\
\text { - } \text { Beschreibung eigener technischer } \\
\text { Medienkompetenz im Vergleich zu } \\
\text { den Eltern } \\
\text { - } \quad \text { Beschreibung eigener Gefühle nach } \\
\text { stundenlangem Medienkonsum } \\
\text { - } \quad \text { Vor- und Nachteile von Geräten im } \\
\text { Zimmer }\end{array}$ & $\begin{array}{l}\text { Medienumgang bewusst } \\
\text { machen } \\
\text { Folgen des Medien- } \\
\text { umgangs erkennen }\end{array}$ \\
\hline
\end{tabular}


Tabelle 6 (fortgesetzt)

\begin{tabular}{|c|c|c|}
\hline Kapitel & Angesprochene Themen & Dimension \\
\hline $\begin{array}{l}\text { Ganz schön } \\
\text { schlimm }\end{array}$ & $\begin{array}{l}\text { - Medienwirkungen: Faszination und } \\
\text { Erfolgserleben } \\
\text { - } \text { Medienwirkungen: Ver- } \\
\text { nachlässigung von Alltagspflichten } \\
\text { und schulischen Aufgaben } \\
\text { - } \quad \text { Medienwirkungen: Angst } \\
\text { - } \quad \text { Weitere Medienwirkungen } \\
\end{array}$ & $\begin{array}{l}\text { Folgen des Medien- } \\
\text { umgangs erkennen }\end{array}$ \\
\hline Bettgeflüster & $\begin{array}{l}\text { - Medienwirkungen: Abhängigkeit } \\
\text { - } \text { Medienwirkungen: Soziale Isolation }\end{array}$ & $\begin{array}{l}\text { Folgen des Medien- } \\
\text { umgangs erkennen }\end{array}$ \\
\hline $\begin{array}{l}\text { Ein schlechtes } \\
\text { Vorbild }\end{array}$ & $\begin{array}{l}\text { - Wahrgenommene Mediennutzung } \\
\text { von Erwachsenen (Eltern, } \\
\text { Lehrer/innen) } \\
\text { - Diskussionsangebot: Der richtige } \\
\text { Umgang mit Fernsehen }\end{array}$ & $\begin{array}{l}\text { Folgen des Medien- } \\
\text { umgangs erkennen } \\
\text { Alternativen entwickeln }\end{array}$ \\
\hline $\begin{array}{l}\text { Leuchttürme und } \\
\text { Lotsen }\end{array}$ & $\begin{array}{l}\text { - Gründe für die Fernsehnutzung: } \\
\text { Langeweile, Gewohnheit } \\
\text { - } \quad \text { Möglichkeiten und Grenzen der } \\
\text { Selbstkontrolle }\end{array}$ & Alternativen entwickeln \\
\hline $\begin{array}{l}\text { Der Zweiwochen- } \\
\text { plan }\end{array}$ & $\begin{array}{l}\text { Einführung des Fernsehplans als } \\
\text { Methode der Selbstregulierung } \\
\text { - Einführung der Aufgabe, beliebte } \\
\text { nicht-mediale Freizeit- } \\
\text { beschäftigungen zu finden und zu } \\
\text { beschreiben }\end{array}$ & Alternativen entwickeln \\
\hline $\begin{array}{l}\text { Brettspiele und } \\
\text { Lerncomputer }\end{array}$ & $\begin{array}{l}\text { - Unterscheidung virtueller Miss- } \\
\text { erfolge und als real wahr- } \\
\text { genommener Misserfolg in Spiel- } \\
\text { situationen } \\
\text { - } \\
\text { - } \\
\text { - Binnvoller Einsatz von Medien } \\
\text { zeitmöglichkeiten }\end{array}$ & Alternativen entwickeln \\
\hline Der Vertrag & $\begin{array}{l}\text { - Einführung des Medienvertrages } \\
\text { - Mögliche Dimensionen eines } \\
\text { Medienvertrages }\end{array}$ & Alternativen entwickeln \\
\hline
\end{tabular}


Begleitend zur Geschichte wurde Lehrkräften eine Mappe mit einem kurzen schriftlichen Leitfaden zur Durchführung des Unterrichts (vgl. Anhang A7) sowie kapitelspezifischen Aufgabenvorschlägen zur Verfügung gestellt (vgl. Anhang A8). Begleitend zur Mappe enthielt das Unterrichtsmanual Kopiervorlagen für ein Medientagebuch (vgl. Anhang A9), sowie eine Elterninformationsbroschüre mit Tipps zur Medienerziehung von Grundschulkindern in deutscher, türkischer und russischer Sprache (Kleimann, Restemeier \& Rehbein, 2006). In einer drei- bis vierstündigen Schulung wurden allen teilnehmenden Lehrkräften während der ersten Stunde empirische Grundlagen zur Mediennutzung von Kindern, vermittelt, insbesondere zum zeitlichen Ausmaß der Mediennutzung, Inhalten der Mediennutzung und ausgewählten Medienwirkungen. Als Datengrundlage dienten dabei die Ergebnisse der KFN-Schülerbefragung 2005 (Mößle et al., 2007). Im Rahmen dieser Schulung wurden den Lehrkräften auch Vorschläge zur Gestaltung von Elternabenden gemacht ${ }^{157}$.

\subsubsection{Konzeptevaluation und Pretest}

Der erste Einsatz des Unterrichtskonzeptes in den Regionen Oldenburg und Soltau-Fallingbostel diente der Evaluation der Unterrichtskonzeption und der formativen Evaluation der Unterrichtseinheit. Dabei wurden die Klassenlehrkräfte $^{158}$ von $\mathrm{N}=21$ zufällig ausgewählten vierten Klassen ${ }^{159}$ im Landkreis

157 Die Elternabende in den Unterrichtsklassen der Berliner Längsschnittevaluation wurden allerdings unter Mitwirkung eines Projektmitarbeiters des KFN gestaltet (vgl. die Ergebnisse des Expertenworkshops zur generellen Wichtigkeit von Elternabenden auf S. 243 dieser Arbeit sowie die Schlussfolgerungen zur Elternbeteiligung nach der formativen Evaluation auf S. 264).

$158 \mathrm{Da}$ den Klassenlehrer/innen in der Grundschule in der Regel ein großes Stundenkontingent in unterschiedlichen Fächern in ihrer Klasse zur Verfügung steht, konnte vermutet werden, dass diese Lehrkräfte am besten in der Lage sein würden, den Medienunterricht in ihren Lehrplan der nächsten Monate integrieren zu können.

159 Die Klassen der eingeladenen Lehrkräfte hatten im Frühjahr 2005 an der KFNGrundschulbefragung 2005 teilgenommen, welcher in Oldenburg eine zufällige Stichprobenziehung vorangegangen war, in Soltau-Fallingbostel war eine Vollerhebung aller vierten Klassen durchgeführt geworden (Baier et al., 2006). Aus dem Pool aller im Frühjahr befragten Schulen wurden für die Erprobung des Präventionskonzeptes in Oldenburg $\mathrm{N}=19$ Klassen und in Soltau-Fallingbostel N=16 Klassen zufällig ausgewählt. Stichprobenausfälle durch grundsätzliche Teilnahmeverweigerungen am Präventionskonzept (Oldenburg N = 8, Absagequote: $42 \%$; Soltau-Fallingbostel N =7, Absagequote: $44 \%$ ) wurden durch Nachrückerklassen ausgeglichen, so dass schließlich in Oldenburg $\mathrm{N}=23$ Klassen aus 10 verschiedenen Schulen ihre Teilnahme zusagten, in Soltau-Fallingbostel $\mathrm{N}=21$ Klassen aus 8 verschiedenen Schulen. 
Soltau-Fallingbostel und von $\mathrm{N}=23$ zufällig ausgewählten vierten Klassen der Stadt Oldenburg zu Workshops ${ }^{160}$ eingeladen, in deren Rahmen sie über zentrale Ergebnisse der KFN-Schülerbefragung 2005 informiert wurden und anschließend um Rückmeldung zum ersten Konzeptionsvorschlag der ersten Stufe des Präventionskonzeptes (Vom Leichtmatrosen zum Medienlotsen) gebeten wurden.

Die Rückmeldungen der beteiligten Lehrkräfte zum Unterrichtskonzept fielen insgesamt sehr positiv aus. Alle Lehrkräfte waren nach Vorstellung des Konzeptes bereit, die Unterrichtseinheit durchzuführen. Die überwiegende Anzahl der an dem Workshop beteiligten Lehrkräfte gab an, den Unterricht in den Fächern Deutsch und Sachkunde geben zu wollen. Trotz der allgemeinen $\mathrm{Zu}$ stimmung zu dem Konzeptvorschlag wurden eine Reihe von Verbesserungsvorschlägen hinsichtlich des Textbuchlayouts gemacht, es wurden einige Hinweise auf schwer verständliche Textpassagen in der Lesegeschichte gegeben sowie hinsichtlich einiger Texte in der Elterninformationsbroschüre. Zudem wurden die Aufgaben- und Fragenvorschläge von einigen Lehrkräften als zu wenig konkret kritisiert. Es wurde allerdings von fast allen Anwesenden die Meinung geäußert, die Aufgaben- und Fragenvorschläge seien eher als Anregung aufzufassen und die konkrete Umsetzung in den Klassen sei dann Aufgabe der Lehrkräfte.

Im Hinblick auf die auf Seite 229 festgelegten Implementationsziele des Unterrichtsprogramms lässt sich festhalten, dass das erste Implementationsziel als erreicht angesehen werden kann: Alle am Workshop beteiligten Lehrkräfte erklären sich bereit, den Unterricht durchzuführen.

Aufgrund der Vorschläge der Lehrkräfte im Workshop wurden Layout und einige Texte der Lesegeschichte, Vorschläge zum zeitlichen Rahmen des Unterrichts sowie inhaltliche und didaktische Details dieser ersten Unterrichtseinheit überarbeitet. Von der Elterninformationsbroschüre wurden Übersetzungen in die türkische und die russische Sprache in Auftrag gegeben, die in beliebiger Anzahl von den Lehrkräften der einzelnen Klassen angefordert werden konnten.

\subsubsection{Formative Evaluation}

In Kapitel 8 wurde als Ziel der formativen Evaluation des entwickelten Medienunterrichtskonzeptes angegeben, die Bewertung der Lehrkräfte insbesondere zu

160 Für beide Regionen wurden je zwei Workshoptermine angeboten, am 8. und 9. November 2005 in Oldenburg sowie am 14. und 15. November 2005 in Bad Fallingbostel. 
Verständlichkeit und Altersangemessenheit des Programms nach ersten Testläufen des Programms einzuholen, um vor der Durchführung des Hauptprogramms sicher gehen zu können, dass die Programmwirksamkeit nicht an diesen Punkten scheitert. Es wurde darüber hinaus darauf hingewiesen, dass subjektive Einschätzungen der Lehrkräfte zur Wirksamkeit des Programms auf ihre Schülerschaft für die tatsächliche Wirksamkeit eines Programms eine durchaus wichtige Rolle spielen können („Pygmalion-Effekt“, vgl. S. 205 in dieser Arbeit). Insofern sollte auch dieser Punkt Gegenstand einer formativen Evaluation während der Testphase des Programms sein.

Die Unterrichtseinheit Vom Leichtmatrosen zum Medienlotsen wurde von Mitte November 2005 bis Mitte Februar 2006 in 44 Klassen in 18 verschiedenen Grundschulen der Regionen Soltau-Fallingbostel und in Oldenburg durchgeführt. Direkt im Anschluss an die Durchführung erfolgte im Februar 2006 eine Befragung aller an der Unterrichtsintervention beteiligten Lehrkräfte in ihren Schulen. Die Lehrkräfte erhielten von geschulten Interviewerinnen oder Interviewern einen 12-seitigen Fragebogen, der nach einer kurzen Instruktion von den Lehrkräften selbst ausgefüllt wurde. Der Fragebogen wurde im Anschluss durch die Interviewerin oder den Interviewer wieder eingesammelt. In 40 der 44 Unterrichtsklassen wurden Fragebögen ausgefüllt (der Fragebogen findet sich im Anhang A10 dieser Arbeit). Da fünf dieser Fragbögen von nicht am Medienunterricht beteiligten Lehrkräften ausgefüllt worden waren, lagen letztlich 35 gültige Lehrkräftefragebögen mit Einschätzungen zum Verlauf und zur Bewertung des Medienunterrichtes vor.

Die befragten Lehrkräfte waren im Durchschnitt 49,9 Jahre alt (SD = 10,2), 31 Lehrkräfte waren weiblichen $(88,6 \%), 4$ Lehrkräfte männlichen Geschlechtes. Gefragt danach, wie gut den Lehrkräften die Klasse bekannt sei, in der der Medienunterricht durchgeführt wurde (3-stufige Skala: $1=$ nicht gut; 2 = eher gut; 3 = sehr gut), gaben 25 Lehrkräfte an, die Klasse sehr gut zu kennen, 10 Lehrkräfte kannten die Klasse eher gut. Im Durchschnitt unterrichteten sie die Klasse seit 36 Monaten $(\mathrm{SD}=11,1), 34$ der Lehrkräfte hatten die Funktion der Klassenlehrkraft inne. 27 Lehrkräfte gaben an, in der betreffenden Klasse unter anderem das Fach Deutsch zu unterrichten, 26 gaben Sachunterricht, 20 dieser Lehrkräfte waren für den Unterricht in beiden Fächern zuständig. Keine der befragten Lehrkräfte war weder für den Deutschunterricht zuständig, noch für den Sachkundeunterricht. Im Durchschnitt gaben die Lehrkräfte an, 13,4 Unterrichtsstunden für den Medienunterricht verwendet zu haben ( $\mathrm{SD}=3,8$; Median: 12 Unterrichtsstunden), es wurden mindestens 7 Unterrichtsstunden gegeben, die längste Unterrichtsdauer betrug 22 Unterrichtsstunden. 
Die Einschätzung der Lehrkräfte zur Medienunterrichtseinheit war nach dem praktischen Einsatz der Unterrichtsmaterialien überwiegend positiv. Auf die Frage, wie gut sie durch die vorangegangene Lehrkräfteschulung auf den Unterricht vorbereitet worden waren (4-stufige Antwortskala: $1=$ sehr gut; $2=$ eher gut; 3 = weniger gut; 4 = gar nicht gut) antworteten 17 Lehrkräfte mit „,sehr gut“, und weitere 15 Lehrkräfte mit „eher gut“. Lediglich zwei Lehrkräfte fühlten sich „weniger gut" auf den Unterricht vorbereitet, eine Person machte keine Angabe. Auf die Frage, wie gut die Geschichte bei den Kindern der Klasse angekommen sei (4-stufige Antwortskala: $1=$ sehr gut; $2=$ eher gut; $3=$ weniger gut; $4=$ gar nicht gut), antworteten 23 Lehrkräfte, die Geschichte sei sehr gut angekommen, die anderen 12 Lehrkräfte gaben an, die Geschichte sei gut angekommen. 34 Lehrkräfte beurteilten die Geschichte nach dem Einsatz im Unterricht als dem Alter der Kinder angemessen, nur eine Lehrkraft befand die Geschichte sei eher für jüngere Kinder geeignet ${ }^{161}$. Die Aufgabenvorschläge, die den Lehrkräften zur Bearbeitung der Lesegeschichte zur Verfügung gestellt worden waren, befanden 17 der befragten Lehrerkräfte als sehr hilfreich, 14 als eher hilfreich und 4 Lehrkräfte als weniger hilfreich (4-stufige Antwortskala: $1=$ sehr hilfreich; $2=$ eher hilfreich; 3 = weniger hilfreich; 4 = gar nicht hilfreich).

Die Lehrkräfte wurden auch danach gefragt, inwieweit sie ihre pädagogische Freiheit bei der Medienerziehung durch die Unterrichtseinheit zu sehr eingeschränkt sahen, oder ob sie sich mehr konkrete Vorgaben zur Durchführung der Unterrichtseinheit gewünscht hätten ${ }^{162}$. Lediglich eine Lehrkraft gab an, sie wäre gerne noch freier in der Planung und Gestaltung des Unterrichtes gewesen, sechs Lehrerinnen beziehungsweise Lehrer hätten sich dagegen mehr konkrete Vorschläge zur Planung und Gestaltung des Unterrichtes gewünscht. 28 Lehrkräfte befanden die Vorschläge zur Gestaltung und Planung des Unterrichtes als gerade richtig. Die Einschätzungen der Befragten, ob die Medienunterrichtseinheit auch längerfristige Wirkung auf das Medienverhalten der Schülerinnen oder Schüler hat, lässt sich als „,vorsichtig optimistisch“ interpretieren: Auf einer vierstufigen Skala $(1=$,ja, auf jeden Fall“; $2=$ „,eher ja; $3=$,, eher nein; $4=$ „nein,

161 Konkrete Fragestellung: „Ist die Geschichte Vom Leichtmatrosen zum Medienlotsen dem Alter der Kinder in Ihrer Klasse angemessen?" Es gab drei Antwortmöglichkeiten: 1.: „Ja, genau richtig.“ 2.: „Nein, die Geschichte ist eher passend für jüngere Kinder.“ 3. : „Nein, die Geschichte ist eher passend für ältere Kinder.“

162 Konkret lautete die Frage: „Die Unterrichtseinheit ist so gestaltet, dass Sie relativ frei in der konkreten Gestaltung des Unterrichtes sind. Wie beurteilen Sie diesen Aspekt?" Es gab drei Antwortmöglichkeiten: 1.: „Ich hätte mir mehr konkrete Vorschläge für die Planung und Gestaltung des Unterrichtes gewünscht.“ 2.: „Ich wäre in meinen Entscheidungen über Planung und Gestaltung des Unterrichtes gerne freier gewesen." 3.: „Ich fand die Vorschläge für Planung und Gestaltung des Unterrichtes gerade richtig.“ 
auf keinen Fall“") antworteten drei Lehrkräfte mit ,,ja, auf jeden Fall“, 25 Befragte mit „eher ja“ und lediglich 6 Lehrkräfte mit „eher nein“. Eine Person machte keine Angaben zu diesem Punkt.

Um einschätzen zu können, inwieweit alle zentralen Elemente der Unterrichtseinheit auch tatsächlich eingesetzt worden waren, wurden die Lehrkräfte konkret nach dem Einsatz der Elemente Lesegeschichte, Fernsehplan, Medienvertrag, Elternbroschüre und Elternabend gefragt. Tabelle 7 zeigt, dass die Lesegeschichte und das Medientagebuch von allen befragten Lehrkräften zumindest in Teilen eingesetzt wurden. Auch der Fernsehplan und die Elterninformationsbroschüre wurden sehr häufig eingesetzt und von lediglich 3 beziehungsweise 2 Lehrkräften gar nicht verwendet.

Tabelle 7: Einsatz der verschiedenen Unterrichtselemente durch die Lehrkräfte $(\mathrm{n}=35$ Unterrichtsklassen, in denen Medienunterricht durchgeführt wurde)

\begin{tabular}{|lllll|}
\hline Unterrichtselement & $\begin{array}{l}\text { komplett ein- } \\
\text { gesetzt }\end{array}$ & $\begin{array}{l}\text { teilweise ein- } \\
\text { gesetzt }\end{array}$ & $\begin{array}{l}\text { nicht ein- } \\
\text { gesetzt }\end{array}$ & $\begin{array}{l}\text { Keine } \\
\text { Angabe }\end{array}$ \\
\hline Lesegeschichte & 34 & 1 & - & - \\
\hline Medientagebuch & 31 & 4 & - & - \\
\hline Fernsehplan & 19 & 13 & 3 & - \\
\hline Medienvertrag & 10 & 10 & 13 & 2 \\
\hline $\begin{array}{l}\text { Elterninforma- } \\
\text { tionsbroschüre }\end{array}$ & 33 & - & 2 & - \\
\hline Elternabend & $20{ }^{163}$ & - & 15 & - \\
\hline
\end{tabular}

Probleme zeigten sich jedoch hinsichtlich der Implementation der Unterrichtselemente Medienvertrag und Elternabend. Diese wurden nur von 20 Lehrkräften eingesetzt, 9 Lehrkräfte setzten hingegen keins der beiden Elemente ein. Vor dem Hintergrund, dass beiden Elementen eine entscheidende Bedeutung im Hinblick auf die Sensibilisierung des Elternhauses und das zukünftige Medienverhalten der Schülerinnen und Schüler zukommt, müssen diese Ergebnisse als unbefriedigend bewertet werden. In den Klassen, in denen Elternabende stattfanden, war die Teilnahmequote an den Elternabenden zudem nicht besonders hoch. Die Lehrkräfte gaben im Schnitt eine Teilnahmequote von 56 Prozent an $(\mathrm{SD}=23,7)$. Dabei zeigte sich, dass bei den fünf Elternabenden, die als zusätz-

$16315 \mathrm{mal}$ als Teil eines Elternabends, $5 \mathrm{mal}$ als Themenelternabend zur Medienerziehung. 
licher Themenelternabend zur Medienerziehung konzipiert waren, die Teilnahmequote mit 31,6 Prozent $(\mathrm{SD}=30,3$ !) deutlich unter der Teilnahmequote jener Elternabende lag, bei denen das Thema Medienerziehung in einen regulären Elternabend integriert wurde (Durchschnittliche Teilnahmequote von $66,3 \%, \mathrm{SD}=9,8)$.

Die Tatsache, dass von neun Lehrkräften weder ein Elternabend durchgeführt wurde, noch ein Medienvertrag erarbeitet wurde, spricht für eine systematische Verknüpfung des Fehlens beider Elemente. Zeitmangel war offensichtlich nicht der Grund für den Verzicht. So zeigt sich, dass Lehrkräfte, die beide Elemente nicht verwendet haben, nicht weniger Zeit für die Unterrichtseinheiten verwendet haben (13,3 Unterrichtsstunden; $\mathrm{SD}=2,8)$, als ihre Kollegen, die beide oder zumindest eins der beiden Elemente einsetzten (13,4 Unterrichtsstunden; $\mathrm{SD}=4,3)$. Auch in der Einschätzung der Wirksamkeit des Unterrichts, der Altersangemessenheit der Unterrichtsinhalte und der Bewertung der Lesegeschichte durch die Schülerschaft gab es keine systematischen Unterschiede zwischen den Gruppen. Auffälligkeiten zeigten sich bei der Gruppe der neun „Verweigerer“ jedoch hinsichtlich der Einschätzungen zur Lehrerschulung und der Qualität des Unterrichtsmaterials. So fanden sich die beiden Lehrkräfte, welche die Lehrerschulung zur Unterrichtseinheit als „weniger gut“ beurteilten, in der Gruppe der Verweigerer, drei Mitglieder dieser Gruppe empfand die Unterrichtsmaterialen als zu wenig konkret. Zudem befanden sich in der Gruppe der „Verweigerer“ überproportional mehr ältere Lehrkräfte. Sechs der neun Lehrkräfte in dieser Gruppe waren 55 Jahre oder älter, nur zwei Lehrkräfte waren jünger als 49 Jahre.

Die Ergebnisse der formativen Evaluation zeigen insgesamt eine gute Akzeptanz der Unterrichtseinheit auf Seiten der Lehrkräfte und - nach Aussagen der Befragten - auch auf Seiten der Schülerinnen und Schüler. Die Qualität der Schulung, die Altersangemessenheit der Inhalte, die Passung zwischen Vorgaben des Konzeptes und pädagogischer Freiheit sowie das Wirkpotential der Unterrichtseinheit wurden vom Durchschnitt der Lehrkräfte als gut eingeschätzt. Probleme zeigten sich bei der Implementierung der Instrumente Medienvertrag und Elternabend. Bezüglich der Verwendung des Medienvertrages wurde für die Umsetzung des Programms im Rahmen des Berliner Längsschnitt Medien die Konsequenz gezogen, dass im Rahmen der Lehrkräfteschulung noch deutlicher auf die Wichtigkeit dieses Instrumentes hingewiesen werden sollte. Da sich im Rahmen der formativen Evaluation zeigte, dass insbesondere ältere Lehrkräfte, die darüber hinaus weniger zufrieden mit der Schulung und den Unterrichtsinhalten waren, dieses Element nicht einsetzten, wurde für die Durchführung des Hauptprogramms beschlossen, im Rahmen des vorangehenden Lehrkräftework- 
shops insbesondere auf die Fragen und Einwände älterer Lehrkräfte einzugehen und zusammen mit den Lehrkräften praktische Beispiele zu Form und Inhalt eines Medienvertrages zu erarbeiten.

Bezüglich der Veranstaltung von Elternabenden wurden drei Schlussfolgerungen gezogen: Erstens erscheint es notwendig, im Rahmen der Lehrkräfteschulung auf die Wichtigkeit dieses Instrumentes hinzuweisen. Zweitens sollte Lehrkräften dringend geraten werden, keinen eigenen Themenelternabend $\mathrm{zu}$ veranstalten, sondern das Thema Medienerziehung in einen regulären Elternabend zu integrieren, da hier eine deutlich höhere Teilnahmequote zu erwarten ist. Drittens sollte - wegen der insgesamt eher mäßigen Teilnahmequote auch an regulären Elternabenden mit dem Thema Medienerziehung - darüber nachgedacht werden, den Elternabend unter Mitwirkung eines externen Experten zu veranstalten, wie dies im Expertenworkshop bereits angeregt wurde (vgl. S. 243 in dieser Arbeit). Dies war im Rahmen des Pretests von Seiten des KFN und der teilnehmenden Schulen nicht zu leisten, wurde aber für die Hauptstudie in Berlin fest eingeplant.

\subsubsection{Durchführung und Evaluation der ersten Unterrichtseinheit}

\subsection{Konzeptänderungen im Vergleich zum Konzepttest in Oldenburg und Soltau-Fallingbostel}

Aufgrund der allgemein positiven Ergebnisse der formativen Evaluation des ersten Unterrichtskonzeptes wurde das Konzept weitgehend unverändert in den 20 Unterrichtsklassen des Berliner Längsschnitt Medien eingesetzt. Dabei wurde im Rahmen der Lehrkräfteworkshops in Berlin, die Mitte Februar 2006 stattfanden, die Wichtigkeit der Elemente Medienvertrag und Elternabend im Vergleich zum Konzepttest in Soltau-Fallingbostel und Oldenburg stärker betont. Bezüglich der Elternabende wurde den Lehrkräften nahegelegt, das Thema der Medienerziehung nicht bei einem reinen Themenelternabend $\mathrm{zu}$ besprechen, sondern im Rahmen eines allgemeinen Elternabends, in dessen Rahmen auch andere finanzielle und organisatorische Entscheidungen $\mathrm{zu}$ treffen waren. Außerdem wurde den Lehrkräften angeboten, dass ein Mitglied des KFNForschungsteams als Experte für kindliche Mediennutzung für einen 30- bis 45minütigen Vortrag im Rahmen eines von den Lehrkräften organisierten Elternabends zur Verfügung steht. 
Am dem Medienunterricht vorangehenden Lehrkräfte-Workshop im Februar 2006 nahmen 16 Lehrkräfte der 20 Unterrichtsklassen teil, den vier Lehrkräften, die aus Krankheitsgründen fehlten, wurden alle Unterlagen schriftlich zugestellt. Zwischen März und Juni 2006 wurde die Medienunterrichtseinheit in 19 der 20 Unterrichtsklassen durchgeführt. Über die Gründe der Nicht-Durchführung der Unterrichtseinheit in einer Klasse können keine Angaben gemacht werden, da die betreffende Lehrkraft den Fragebogen für Lehrkräfte nicht ausfüllte und im direkt folgenden Schuljahr die Klassenleitung der betreffenden Klasse wechselte. In 17 der 19 Unterrichtsklassen fanden Elternabende unter Beteiligung eines Mitglieds des KFN-Forschungsteams statt. In zwei Klassen wurden von den Lehrkräften keine Elternabende organisiert.

\subsection{Evaluation der Programmdurchführung}

Die Evaluation der Programmdurchführung fand in Form einer Befragung der Lehrkräfte der Unterrichtsklassen zur Implementation des Medienunterrichtes statt. In Kapitel 8 wurde bereits dargelegt, dass Implementationsforschung während der Programmdurchführung Anhaltspunkte über mögliche Probleme in der Programmimplementation liefern kann und insbesondere solche Sachverhalte begleitend zur Programmdurchführung erfasst werden sollten, von denen angenommen werden kann, dass sie intervenierende Größen im Rahmen der Programmwirksamkeitsevaluation darstellen.

Die Unterrichtseinheit wurde in den teilnehmenden Klassen zwischen Februar 2006 und Juni 2006 durchgeführt. Die Befragung der N = 19 Lehrkräfte, in deren Klassen die Medienunterrichtseinheit Vom Leichtmatrosen zum Medienlotsen durchgeführt wurde, fand im Rahmen des zweiten Messzeitpunktes ( $\mathrm{t} 2$; Mai/Juni 2006) des Berliner Längsschnitt Medien statt. Von allen $\mathrm{N}=19$ Lehrkräften wurden gültige Fragebögen ausgefüllt ${ }^{164}$.

164 In einem Fall wurde der Unterricht zum Teil von einem Lehramtsreferendar ausgeführt, der Fragebogen aber von der Klassenlehrkraft ausgefüllt. Da der Unterricht des Referendars von dieser Lehrkraft regelmäßig besucht und beaufsichtigt wurde, wurde auch dieser Fragebogen in die Auswertung mit einbezogen. Von der Lehrkraft, in deren Klasse der Unterricht nicht durchgeführt wurde, wurde auch kein Lehrkräftefragebogen ausgefüllt, so dass Rückschlüsse auf die Gründe der Nichtdurchführung des Programms nicht möglich sind. 
Die befragten Lehrkräfte waren im Durchschnitt 46,4 Jahre alt (SD = 7,6), 17 Lehrkräfte waren Frauen (89,5 \%), 2 Lehrkräfte Männer. Neun der Befragten gaben an, die Klasse, in der der Medienunterrichts durchgeführt wurde, ,sehr gut“ zu kennen, zehn Befragte kannten die Klasse „eher gut“ (3-stufige Skala: $1=$ nicht gut; $2=$ eher gut; $3=$ sehr gut), wobei die Lehrkräfte die Klasse im Durchschnitt seit 27 Monaten (SD = 11,3) unterrichteten. Alle den Medienunterricht ausführenden Lehrkräfte waren zugleich Klassenlehrkraft in der Klasse. Alle 19 befragten Lehrkräfte gaben an, in der betreffenden Klasse unter anderem das Fach Deutsch zu unterrichten, 18 Lehrkräfte gaben auch Sachunterricht. Im Durchschnitt wurden 14,1 Unterrichtsstunden für den Medienunterricht verwendet ( $\mathrm{SD}=5,3$; Median: 7 Unterrichtsstunden), wobei mindestens 5 Unterrichtsstunden stattfanden. Die längste Unterrichtdauer betrug 26 Unterrichtsstunden. Damit lag das verwendete Stundenkontingent in etwa auf dem Niveau des Konzepttests in Oldenburg und Soltau-Fallingbostel, wo durchschnittlich 13,4 Unterrichtsstunden benötigt wurden.

Die Einschätzung der Lehrkräfte zur Medienunterrichtseinheit war nach dem praktischen Einsatz der Unterrichtsmaterialien durchaus positiv, wobei nicht von allen Lehrkräften Antworten zu allen Teilbereichen des Unterrichtspaketes gemacht wurden. Von den 15 Lehrkräften, die am Lehrerworkshop zur Vorbereitung der Unterrichtseinheit teilgenommen hatten, beurteilten 9 den Workshop als ,sehr gut", 5 beurteilten ihn als eher gut, eine Lehrkraft befand ihn als „weniger gut“ (4-stufige Antwortskala: $1=$ sehr gut; $2=$ eher gut; $3=$ weniger gut; 4 = gar nicht gut). 12 Lehrkräfte gaben an, die Lesegeschichte Vom Leichtmatrosen zum Medienlotsen sei „sehr gut“ bei ihren Schülerinnen und Schülern angekommen, 6 schätzen die Akzeptanz der Geschichte bei den Kindern mit „eher gut“ ein, eine Lehrkraft mit „weniger gut“ (4-stufige Antwortskala: $1=$ sehr gut; 2 = eher gut; 3 = weniger gut; 4 = gar nicht gut). Damit lag die von den Lehrkräften wahrgenommene Akzeptanz der Geschichte bei den Kindern in etwa auf dem Niveau des Konzepttests in Oldenburg und Soltau-Fallingbostel. 18 Lehrkräfte befanden die Geschichte für ihre Schülerschaft als altersangemessen, eine Lehrkraft befand die Geschichte sei eher für ältere Kinder geeignet $^{165}$. Dies entspricht in etwa der Einsätzung der Lehrkräfte beim Konzepttest in Soltau-Fallingbostel und Oldenburg und zeigt, dass die Geschichte aus Lehrkräftesicht sowohl in dritten wie auch in vierten Klassen angewendet

165 Konkrete Fragestellung: „Ist die Geschichte Vom Leichtmatrosen zum Medienlotsen dem Alter der Kinder in Ihrer Klasse angemessen?" Es gab drei Antwortmöglichkeiten: 1.: „Ja, genau richtig.“ $2 .:$ „Nein, die Geschichte ist eher passend für jüngere Kinder.“ 3 .: „Nein, die Geschichte ist eher passend für ältere Kinder.“ 
werden kann. Die vorbereiteten Fragen und Aufgabenvorschläge, befanden 6 der befragten Lehrerinnen und Lehrer als sehr hilfreich, 8 als eher hilfreich und 5 Lehrkräfte als weniger hilfreich (4-stufige Antwortskala: 1=sehr hilfreich; 2 = eher hilfreich; 3 = weniger hilfreich; $4=$ gar nicht hilfreich), womit die Einschätzung der Berliner Lehrkräfte kritischer ausfielt als die Einschätzung der Lehrkräfte im Konzepttest.

Die Frage nach der Angemessenheit des Unterrichtsmaterials bezüglich der Handlungsfreiheit der Lehrkräfte ${ }^{166}$ wurde wie folgt beantwortet. Fünf Befragte hätten sich mehr konkrete Vorschläge zur Planung und Gestaltung des Unterrichtes gewünscht, 14 Lehrkräfte befanden die Vorschläge zur Gestaltung und Planung des Unterrichtes als gerade richtig. Damit fiel auch diese Einschätzung, obschon insgesamt deutlich positiv, etwas kritischer aus als beim Konzepttest. Ähnliches zeigte sich bei der Einschätzung der Berliner Lehrkräfte bezüglich der längerfristigen Wirkung der Unterrichtseinheit. Auf der vierstufigen Skala $(1=$, ja, auf jeden Fall“; $2=$ „,eher ja; $3=$,, eher nein; $4=$ „,nein, auf keinen Fall“") antworteten zwei befragte mit ,ja, auf jeden Fall“, zehn Befragte mit „eher ja“ und 6 Lehrkräfte mit „eher nein“. Eine Person machte keine Angaben zu diesem Punkt.

Auch im Berliner Längsschnitt Medien wurden die Lehrkräfte nach dem Einsatz der zentralen Elemente der Unterrichtseinheit gefragt, und gebeten, Angaben über die Verwendung der Elemente Lesegeschichte, Fernsehplan, Medienvertrag, Elternbroschüre und Elternabend zu machen (vgl. Tabelle 8, S. 268). Es zeigte sich, dass die Lesegeschichte und das Medientagebuch von allen befragten Lehrkräften zumindest in Teilen eingesetzt wurden, auch die Elternbroschüre wurde von allen Lehrkräften, die Angaben zu diesem Punkt machten, verteilt. Dieses Ergebnis entspricht in etwa dem des Konzepttests in Oldenburg und Soltau-Fallingbostel. Hinsichtlich des Fernsehplans zeigte sich in Berlin eine leicht geringere Durchführungsquote als beim Konzepttest, wobei 15 Lehrkräfte angaben, das Instrument zumindest teilweise eingesetzt zu haben.

166 Konkret lautete die Frage: „Die Unterrichtseinheit ist so gestaltet, dass Sie relativ frei in der konkreten Gestaltung des Unterrichtes sind. Wie beurteilen Sie diesen Aspekt?" Es gab drei Antwortmöglichkeiten: 1.: „Ich hätte mir mehr konkrete Vorschläge für die Planung und Gestaltung des Unterrichtes gewünscht.“ 2.: „Ich wäre in meinen Entscheidungen über Planung und Gestaltung des Unterrichtes gerne freier gewesen." 3.: „Ich fand die Vorschläge für Planung und Gestaltung des Unterrichtes gerade richtig.“ 
Tabelle 8: Einsatz der verschiedenen Unterrichtselemente durch die Lehrkräfte in $\mathrm{N}=19$ Unterrichtsklassen, in denen Medienunterricht durchgeführt wurde

\begin{tabular}{|lllll|}
\hline Unterrichtselement & $\begin{array}{l}\text { komplett ein- } \\
\text { gesetzt }\end{array}$ & $\begin{array}{l}\text { teilweise ein- } \\
\text { gesetzt }\end{array}$ & $\begin{array}{l}\text { nicht ein- } \\
\text { gesetzt }\end{array}$ & $\begin{array}{l}\text { Keine } \\
\text { Angabe }\end{array}$ \\
\hline Lesegeschichte & 17 & 2 & - & - \\
\hline Medientagebuch & 16 & 2 & - & 1 \\
\hline Fernsehplan & 8 & 7 & 3 & 1 \\
\hline Medienvertrag & 3 & 7 & 8 & 1 \\
\hline $\begin{array}{l}\text { Elterninforma- } \\
\text { tionsbroschüre }\end{array}$ & 18 & - & - & 1 \\
\hline Elternabend & $17^{167}$ & - & 2 & - \\
\hline
\end{tabular}

Der Medienvertrag wurde in Berlin, wie bereits beim Konzepttest in Norddeutschland, verhältnismäßig wenig eingesetzt, wobei der Einsatz in Berlin noch nicht einmal an das Niveau beim Konzepttest heranreichen konnte. Lediglich drei Lehrkräfte führten dieses Unterrichtselemente komplett durch, sieben zumindest in Ansätzen. Dies zeigt, dass die Bemühungen, innerhalb des Lehrkräfteworkshops die Wichtigkeit dieses Elementes zu betonen, nicht erfolgreich waren. Über konkrete Gründe, warum der Medienvertrag nur relativ wenig eingesetzt wurde, lässt sich allerdings nur spekulieren. Die im Rahmen der formativen Evaluation herausgearbeiteten Einflussfaktoren auf die weniger konsequente Umsetzung dieses Unterrichtselementes zeigten sich im Rahmen der Berliner Studie weniger deutlich. So waren die Lehrkräfte, die den Medienvertrag nicht einsetzten, weder älter als ihre Kolleginnen und Kollegen, noch waren sie weniger zufrieden mit dem Einführungsworkshop, oder den vorbereiteten Arbeitsblättern. Allerdings hätten sich drei Lehrkräfte, die den Medienvertrag nicht einsetzen, mehr konkrete Vorschläge zur Durchführung des Unterrichtes gewünscht, während fünf Lehrkräfte die Vorschläge für gerade richtig befand. In der Gruppe derer, die den Medienvertrag einsetzen, war die Beurteilung der Konkretheit der Vorschläge deutlich höher ( 9 fanden die Vorschläge gerade richtig konkret, lediglich 2 hätten sich konkretere Vorschläge gewünscht). Zudem zeigten sich die Lehrkräfte, die den Medienvertrag nicht einsetzten, deutlich skeptischer im Hinblick auf die längerfristige Wirkung des Unterrichts. Von den sieben Lehrkräften dieser Gruppe, die die Frage nach der

1679 mal als Teil eines Elternabends, 8 mal als Themenelternabend zur Medienerziehung. 
längerfristigen Wirksamkeit des Unterrichts beantworteten, gaben drei Lehrkräfte an, der Unterricht habe eher längerfristige Wirkungen, vier dagegen sagten, der Unterricht habe eher keine längerfristigen Wirkungen.

Im Gegensatz zum Medienvertrag, der im Vergleich zum Konzepttest nicht häufiger eingesetzt wurde, konnte die Quote durchgeführter Elternabende und die Teilnahme an diesen Elternabenden im Gegensatz zum Konzepttest deutlich gesteigert werden, wobei sich insbesondere die Mitwirkung eines externen Experten positiv ausgewirkt haben dürfte. In 17 von 19 Klassen wurden Elternabende unter Mitwirkung eines externen Experten zur Medienerziehung durchgeführt, die Teilnahmequote lang nach Einschätzung der Lehrkräfte bei durchschnittlich 65,2 Prozent (SD =22,3), wobei die im Rahmen des Workshops kommunizierte Strategie, den Elternabend nicht als reinen Themenelternabend zur Medienerziehung zu konzipieren, nur von 9 Lehrkräften umgesetzt wurde. Die 8 Lehrkräfte, die zu einem Themenelternabend Medienerziehung einluden, gaben an, dass zum Zeitpunkt des Elternabends keine weiteren organisatorischen oder inhaltlichen Themen mit den Eltern zu besprechen gewesen seien. Dabei zeigt sich auch im Rahmen des Berliner Längsschnitt Medien, dass bei den 8 Elternabenden, die als reiner Themenelternabend zur Medienerziehung konzipiert waren, die Teilnahmequote mit 56,6 Prozent $(\mathrm{SD}=18,6)$ deutlich unter der Teilnahmequote jener Elternabende lag, bei denen das Thema Medienerziehung in einen regulären Elternabend integriert wurde (Durchschnittliche Teilnahmequote von $72,8 \%, \mathrm{SD}=23,6$ ).

Die Ergebnisse der Evaluation der Durchführung der ersten Medienunterrichtseinheit zeigen insgesamt eine recht gute Akzeptanz der Unterrichtseinheit auf Seiten der Lehrkräfte und - nach Aussagen der Pädagogen - auch auf Seiten der Schülerinnen und Schüler, wobei die Unterrichtseinheit insgesamt etwas skeptischer aufgenommen wurde als beim Konzepttest in Norddeutschland. Als positives Resultat der Konsequenzen der formativen Evaluation konnte die Teilnahmequote an den Elternabenden deutlich gesteigert werden, der Medienvertrag wurde jedoch nur in unzureichendem Maß umgesetzt. 


\subsubsection{Das zweite Unterrichtskonzept (4. Klassen)}

Im Rahmen der ersten Unterrichtseinheit Vom Leichtmatrosen zum Medienlotsen (vgl. S. 248 - 258) wurden die Schülerinnen und Schüler für die Bedeutung elektronischer Medien in ihrem Alltag sensibilisiert. Gleichzeitig fand ein Dialog zwischen Kindern und Lehrkräften über den Medienalltag der Kinder statt, so dass die Lehrkräfte in der Lage waren, auf dieser Basis zusammen mit ihrer Schülerschaft Konzepte zur Erfassung des Medienalltags (Medientagebuch), zur Planung der Mediennutzung (Fernsehplan) und zur ausgewogenen Freizeitgestaltung (Medienvertrag) zu erarbeiten.

Bei der Konzeption einer nachfolgenden Unterrichtseinheit galt es zunächst zu entscheiden, inwieweit die mit den Schülerinnen und Schülern bisher erarbeiteten Inhalte und Verhaltensweisen im Sinne einer „booster session“ (vgl. S. 184 in dieser Arbeit) lediglich verstärkt werden sollten, oder ob eine nachfolgende Unterrichtseinheit andere Schwerpunkte bei der Auseinandersetzung mit problematischen Mediennutzungsmustern und ihrer Prävention setzen sollte. Letztlich wurde entschieden, eine Unterrichtseinheit mit neuen thematischen Schwerpunkten zu erarbeiten. Vor dem Hintergrund, dass bereits in der vierten Klasse und insbesondere bei männlichen Schülern problematische Mediennutzungsmuster nachgewiesen wurden, von denen anzunehmen ist, dass sie sich zumindest bei einigen Schülern im Jugendalter verfestigen (vgl. Kapitel 6 in dieser Arbeit), sollte die zweite Unterrichtseinheit vor allem problematische Mediennutzungsmuster, ihre Entstehungshintergründe und ihre Wirkungen, stärker in den Blick zu nehmen. Auf den Seiten 193 - 195 dieser Arbeit wurde bereits dargestellt, dass Kinder im späten Grundschulalter durchaus in der Lage sind, kausale Beziehungen zwischen problematischen Mediennutzungsmustern und ihren Wirkungen zu verstehen. Es wurde aber auch gezeigt, dass ein grundsätzliches Verständnis dieser kausalen Beziehungen gerade bei Minderjährigen mit bereits problematischen Mediennutzungsmustern keineswegs zu einer Verhaltensänderung führen muss, da ihr Verhalten von ihnen selbst als durchaus funktional wahrgenommen werden kann (vgl. S. 156 - 158 in dieser Arbeit), da sie aus ihrem sozialen Umfeld, insbesondere der Peergroup, in ihrem Verhalten teilweise eher gestützt als in Frage gestellt werden (vgl. S. 129 in dieser Arbeit), und da ihnen häufig ein Verständnis für negative Auswirkungen fehlt, wenn diese sich erst mittel- oder langfristig zeigen. Insofern erschien es bei der Konzeption der zweiten Unterrichtseinheit besonders wichtig, nicht nur die Schülerinnen und Schüler - insbesondere jene mit bereits problematischer Mediennutzung - auf die potentiellen Risiken ihres Medienhandelns aufmerksam zu machen, sondern auch ihr Umfeld - Peers, Lehrkräfte und Eltern - für die 
Risiken problematischer Mediennutzung zu sensibilisieren und Anzeichen einer solchen problematischen Mediennutzung zu erkennen. Auch die zweite Unterrichtseinheit wurde nach dem Dreischritt „Medienumgang bewusst machen“ „Folgen des Medienumgangs erkennen“ - „Alternativen erkennen“ aufgebaut (vgl. Abbildung 4, S. 255) wobei die Lesegeschichte insbesondere die Folgen problematischen Medienumgangs thematisiert.

\subsubsection{Unterrichtsmaterial}

Aufgrund der positiven Rückmeldungen der Berliner Lehrkräfte zum Instrument der Lesegeschichte als Zentrum medienerzieherischer Aktivitäten im Unterricht, wurde auch für die zweite Unterrichtseinheit eine Lesegeschichte entwickelt (zu den Vorteilen dieses Instrumentes vgl. S. 248 - 250 in dieser Arbeit). Auch dieses Mal sollte gewährleistet sein, dass die Unterrichtseinheit sowohl in den Fächern Deutsch als auch im Sachkundeunterricht eingesetzt werden kann, um den Lehrkräften größtmögliche Flexibilität in der zeitlichen Planung ihres Unterrichts zu ermöglichen. Das Thema der zweiten Unterrichtseinheit erforderte jedoch neue Überlegungen zur Konzeption der Lesegeschichte. Kinder mit problematischer Mediennutzung mithilfe einer Lesegeschichte direkt zu einer Verhaltensänderung zu bewegen, wurde von Anfang an als wenig erfolgversprechend angesehen. Daher sollte es primäres Ziel dieser Unterrichtseinheit sein, (noch) nicht betroffene Schülerinnen und Schüler im Rahmen der Unterrichtseinheit für die Existenz solcher problematischer Mediennutzungsmuster zu sensibilisieren, um sich einerseits selbst vor der Entwicklung solcher Mediennutzungsmuster zu schützen und um andererseits frühzeitig zu erkennen, wenn Kinder in der Klasse oder im Freundeskreis Medien in problematischer Weise nutzen. Auf diese Weise sollte erreicht werden, dass Kinder mit problematischen Mediennutzungsmustern von den Peers in der Klasse keine Unterstützung für ihr Verhalten finden, sondern durch das Feedback ihrer Mitschüler für eine Änderung ihres Verhaltens motiviert werden.

Um dies $\mathrm{zu}$ erreichen, musste gewährleistet sein, dass problematische Mediennutzung in der Lesegeschichte als ein Verhaltensmuster dargestellt wird, von dem unter Umständen jedes Kind und jeder Jugendliche betroffen sein kann. Die Schülerinnen und Schüler sollten sich bewusst werden, dass problematisches Medienverhalten spezifische Ursachen hat und dass Betroffene Unterstützung dabei brauchen, ihr Medienverhalten zu ändern. Gleichzeitig sollten auch Lehrkräfte durch die Geschichte sensibilisiert werden, frühzeitig Anzeichen 
problematischer Mediennutzung bei ihren Schülerinnen und Schülern zu erkennen.

Vor dem Hintergrund dieser Überlegungen wurde eine Geschichte entwickelt, deren Hauptfigur sich aufgrund familiärer Probleme immer mehr in die Welt eines Computerspiels flüchtet. Der sympathische Charakter der Hauptfigur und eine detaillierte Darstellung seiner Handlungsmotive macht es den Leserinnen und Lesern leicht, sich mit der Figur zu identifizieren, seine Flucht in die Welt seines Computerspiels wird jedoch stets als problematisch beschrieben.

Konkret wird in der Lesegeschichte eine einzige Nacht im Leben eines Dreizehnjährigen mit dem Namen Bela geschildert. Nur einige Jahre älter als die Zielgruppe der Geschichte, bietet Bela durchaus Identifikationspotential für Leserinnen und Leser im Grundschulalter. Belas Leben ist zum Zeitpunkt der Handlung geprägt von großen Belastungen. Im Mittelpunkt seiner Aufmerksamkeit steht die Sorge um seine Mutter, die im Krankenhaus liegt, um an einem Krebsgeschwür operiert zu werden (vgl. Tabelle 9, S. 274, Textbeispiel 1). Da der Vater die Mutter ins Krankenhaus begleitet hat, ist Bela für einige Tage unter der Aufsicht einer Nachbarin allein in der elterlichen Wohnung. Neben der Sorge um die Mutter und der nächtlichen Einsamkeit in der Wohnung hat Bela auch mit schulischen Problemen zu kämpfen. Seine Noten sind in letzter Zeit - wohl auch aufgrund der Belastungen in der Familie - massiv abgerutscht. Doch seinem strengen und unnahbaren Deutschlehrer kann oder will sich Bela nicht anvertrauen.

Auch im Freundeskreis gibt es Probleme. Bela, der in letzter Zeit in seiner Freizeit fast ausschließlich ein Fantasyspiel ${ }^{168}$ am Computer spielt (vgl. Tabelle 9, S. 274, Textbeispiele 1 und 2), hat sich von seinen alten Freunden in letzter Zeit immer weiter entfernt. Die beiden Freunde teilen Belas Leidenschaft für das Computerspiel nur bedingt und sind außerdem fest in die zahlreichen Freizeitaktivitäten ihrer Familien eingebunden. Auch diese offene Zurschaustellung des intakten Familienlebens seiner Freunde belastet Bela, der sich von seinen Eltern aufgrund der Erkrankung seiner Mutter in letzter Zeit wenig beachtet fühlt (vgl. Tabelle 9, Textbeispiel 4).

So sitzt Bela zum Anfang der Geschichte noch spät am Abend vor dem Computer seines Vaters, spielt allein sein Computerspiel und arbeitet zeitgleich an einem Aufsatz über den gebürtigen Berliner Informatiker Joseph Weizenbaum, dessen Arbeiten in den sechziger Jahren die Forschungen zur Künstlichen Intelligenz prägten. Durch den Aufsatz wird Bela auf das von Weizenbaum ent-

168 Ein erfundenes Computerspiel, welches als eine Mischung aus den Computerspielen Warcraft und World of Warcraft konzipiert ist. 
wickelte Computerprogramm ELIZA aufmerksam. ELIZA wurde bereits in den sechziger Jahren programmiert um zu zeigen, wie mittels eines Computerprogramms ein menschlicher Gesprächspartner simuliert werden kann. Über einen Link im Internet gelangt Bela zu einer modernen Version von ELIZA und fängt an, sich spaßeshalber mit ihr zu unterhalten. Obwohl er fasziniert von der Echtheit einiger Antworten von ELIZA ist, erkennt er relativ schnell die Grenzen dieses Computerprogramms. ELIZA antwortet oftmals schematisch, wiederholt sich oder bezieht sich in ihren Antworten nicht auf das vorher Gesagte. Schließlich schläft Bela, erschöpft vom Arbeiten und dem langen Spielen am Computer, am Schreibtisch ein.

Als Bela nach einem Albtraum erwacht, sieht er, dass ELIZA ihm auf seine letzte Frage anders geantwortet hat als vorher. Die Antwort wirkt menschlich. Und tatsächlich: Im darauf folgenden Gespräch verhält sich ELIZA wie ein menschlicher Gesprächspartner. Bela berichtet ELIZA von seinen zahlreichen Problemen, von seiner Einsamkeit und von seiner Leidenschaft für das FantasyComputerspiel. Daraufhin zeigt ELIZA ihm Gespräche mit zwei anderen Jugendlichen, die sie protokolliert hat: Die ELIZA-Protokolle von Fiona und Mario. In diesen Gesprächen wird deutlich, warum Medien für Kinder und Jugendliche wichtig sein können, aber auch, welche Probleme die Abhängigkeit von diesen Medien unter Umständen mit sich bringt (vgl. Tabelle 9, Textbeispiel 5). Zum ersten Mal seit langer Zeit fühlt sich Bela nicht mehr einsam und unverstanden. 


\section{Textbeispiel 1: Flucht ins Spiel}

Wieder schweifen seine Gedanken ab zu seinen Eltern, zu seiner Mutter, die in irgendeinem Krankenhaus weit weg in Österreich liegt und $\mathrm{zu}$ seinem Vater, der schweigend neben ihrem Bett sitzt und sich Sorgen macht. Immer macht er sich Sorgen... Dann ist Bela mittendrin. Schwerter klirren, Metall trifft auf Metall, Holz auf Holz, und überall schreien Feinde und Verbündete durcheinander. [...] Bela hält kurz inne und klickt sich dann in eine seiner Kasernen, um neue Truppen zu rekrutieren. Es dauert mehr als eine Stunde, bis er den Feind endlich besiegt hat und auch das letzte Gebäude der Orks zerstört ist.

\section{Textbeispiel 2: Selbstwirksamkeit}

„In diesen Spielen kann ich jemand ganz anderes sein. Ich bin kein dreizehnjähriger Schüler mehr, kein normales Kind mit normalen Eltern und einem normalen Leben. Ich kann ein Zauberer oder ein Elfenkrieger sein. Oder auch ein Ork, ein Troll oder ein Kobold. Ich trage Rüstungen, die leicht sind wie Seide und hart wie Diamanten. Ich bin ein Meister aller Waffen, kann mit Schwert, Bogen und Speer umgehen und bin stärker als alle anderen. [...] Die Welt ist anders dort, mit riesigen Wäldern und großen, uneinnehmbaren Burgen. Sie ist voller Magie. [...] Ich kann Verwundete heilen und Kobolde verfluchen. Ich kann einen mächtigen Eisregen beschwören und ihn gegen eine feindliche Armee schicken. Das ist nicht die Wirklichkeit. Es ist viel besser! Wie ein Märchen, in dem man selber mitspielt." 


\section{Textbeispiel 3: Einsamkeit}

Der Bildschirmschoner wird aktiviert, hunderte kleine Lichtpunkte fliegen ihm entgegen und verschwinden am Rand des Monitors. Wie spät ist es? 3:45 Uhr. [Bela] steht kurz auf, und tastet sich langsam durch die Dunkelheit bis zur Kommode mit den Teelichten. Er nimmt die Tüte mit den Kerzen und den Streichhölzern heraus und geht zurück zum Schreibtisch. Das Streichholz flackert auf, als er es am rauen Rand der Schachtel reibt und dann nochmals, als er zwei Teelichte entzündet. Er stellt sie rechts und links des Bildschirms auf und setzt sich wieder vor die Tatstatur. Dann wartet er. Bela ist alleine. Nie hat er sich einsamer gefühlt als gerade jetzt.

\section{Textbeispiel 4: Abschottung}

„[Ich] lasse mir weder von Lukas, von meinem Lehrer noch von einem verdammten Computerprogramm erzählen, dass ich zu viel Computer spiele! Warum denn nicht? Was ist so schlimm daran, dass ich einmal gut in etwas bin? Soll ich den ganzen Tag hier sitzen und mir Sorgen machen? Soll ich abends um acht ins Bett gehen, nur damit ich die ganze Nacht Albträume habe? Soll ich nachmittags irgendwelchen Schwachsinn mit meinen Freunden und ihren ach-sotollen Eltern unternehmen, damit alle mir zeigen können, dass bei ihnen alles in Ordnung ist und bei mir nicht? [...] Computerspielsüchtig, ha! Ich würde manchmal am liebsten in mein Spiel hineinkriechen und nie wieder herauskommen! Wer weiß, kann ja sein, dass mich jemand vermisst. Du hast doch keine Ahnung, ELIZA! Ihr wisst doch alle nichts über mich.“ 


\section{Textbeispiel 5: Fiona}

„Ich kann es sehen, ELIZA, jeden Tag im Fernsehen kann ich sehen, wie die Schauspielerinnen aussehen, wie die Leute in der Werbung aussehen und wie die Nachrichtensprecherinnen aussehen! Und auch in den CastingShows: Hast du schon mal gesehen, wer da in die nächste Runde kommt? [...] Ich kann dir sagen, wer da NICHT weiterkommt: Die fetten Mädchen, die fliegen raus! Letztens haben sie eine dicke Frau gezeigt. Gut, die hat nicht wirklich super gesungen, aber weißt $\mathrm{du}$, was die im Fernsehen gemacht haben? Bei jedem Schritt, den sie gegangen ist, haben die mit der Kamera gewackelt, als wenn die Erde bebt! Und während sie gesungen hat, haben die das Trompeten eines Elefanten eingespielt! Und meine Eltern? Sie haben gelacht!!! Meine eigenen Eltern haben darüber gelacht! Und als ich wütend geworden bin, haben sie gesagt, dass ich keinen Humor habe."

\section{Textbeispiel 6: Albtraum}

Das Telefon klingelt, doch Bela lässt es läuten. $\mathrm{Zu}$ aufgewühlt ist er, um einen klaren Gedanken zu fassen. [...] Panisch rennt Bela aus dem Arbeitszimmer und tastet sich durch den dunklen, kalten Flur. Wieder klingelt das Telefon. Er muss dringend jemanden finden. Immer wieder der gleiche Flur. Er muss sich beeilen, sonst ist es zu spät, doch er kann sich nicht bewegen. Wie angewurzelt steht er in der Mitte des Ganges, und niemand ist da, um ihm zu helfen. Unten aus dem Treppenhaus kommen Schritte. Marschierende Stiefel. Raue Stimmen erklingen, das Klirren rostiger Säbel: Orks! Hunderte! Wo sind seine Waffen? Irgendwo in der Nähe zischt eine Kerze und erlischt. Bela schreit auf und schreckt hoch. Das Telefon klingelt weiter und weiter. Schlaftrunken und verstört nimmt er schließlich ab. Es ist seine Mutter.

Doch dann passiert etwas Seltsames: Die verständnisvolle ELIZA verwandelt sich. Im Gespräch über die in den Protokollen angesprochenen Probleme entpuppt sie sich als größenwahnsinniges Computerprogramm, das den Menschen seine Lösungen aufzwingen will. Auch für Bela hat sie schnell eine Lösung parat: Sein Computerspiel muss schnellstmöglich gelöscht werden. Belas Protest wischt sie barsch beiseite. Dann beginnt sie mit der Umsetzung ihres Plans: Erst kontrolliert ELIZA Belas Computer, später dringt sie sogar in die Telefonanlage ein. Bela gerät in Panik und die ganze Szenerie gewinnt mehr und mehr albtraumhafte Züge. Das Telefon klingelt. Dann erwacht Bela (vgl. Tabelle 9, Textbeispiel 6). Die siebte Szene der Geschichte beschreibt ein letztes Gespräch 
zwischen Bela und ELIZA. Es wird deutlich, dass die ELIZA, die sich zunächst wie ein echter Mensch verhielt und sich nach und nach in ein Monster verwandelte, nur einem Traum von Bela entsprungen ist. Bela berichtet der echten, der Computer-ELIZA, dass seine Eltern wieder zuhause sind und seine Mutter wieder gesund wird. Alles scheint sich zum Guten zu wenden.

Tabelle 10: Aufbau und Themen der Unterrichtseinheit

\begin{tabular}{|c|c|c|}
\hline Kapitel & Inhalt & Angesprochene Themen \\
\hline 1. Nachtschicht & $\begin{array}{l}\text { Einführung der Hauptfigur } \\
\text { Bela }\end{array}$ & $\begin{array}{ll}\text { - } & \text { Sorgen und Probleme im } \mathrm{Zu}- \\
& \text { sammenleben der Familie } \\
\text { - } & \text { Einsamkeit } \\
\text { - } & \text { Schulprobleme } \\
\text { - } & \text { Verwenden von Internetseiten wie } \\
& \text { wikipedia.de für die Hausaufgaben }\end{array}$ \\
\hline $\begin{array}{l}\text { 2. Ein Gespräch } \\
\text { in der Dunkel- } \\
\text { heit }\end{array}$ & $\begin{array}{l}\text { Einführung des Computer- } \\
\text { programms } E L I Z A\end{array}$ & $\begin{array}{ll}\text { - } & \text { Faszination und Grenzen künstlicher } \\
& \text { Intelligenz } \\
\text { - } & \text { Faszination von Computerspielen } \\
\end{array}$ \\
\hline $\begin{array}{l}\text { 3. Ich bin ein } \\
\text { Meister aller } \\
\text { Waffen }\end{array}$ & $\begin{array}{l}\text { Belas Gespräch mit ELIZA } \\
\text { über seine Sorgen und die } \\
\text { Faszinationskraft von } \\
\text { Computerspielen }\end{array}$ & $\begin{array}{l}\text { - } \text { Konflikte im Freundeskreis } \\
\text { - } \text { Computerspielsucht } \\
\text { - Einsamkeit }\end{array}$ \\
\hline 4. Fiona & $\begin{array}{l}\text { Protokoll eines Gesprächs } \\
\text { zwischen ELIZA und } \\
\text { einem Mädchen namens } \\
\text { Fiona über Handys, SMS, } \\
\text { Chatten, Casting-Shows } \\
\text { und über den Fluch, nicht } \\
\text { dem klassischen Schön- } \\
\text { heitsideal zu entsprechen }\end{array}$ & $\begin{array}{l}\text { - Umzug in eine andere Stadt (neue } \\
\text { Klasse, Verlust der alten Freunde) } \\
\text { - } \quad \text { Neue Medien als Instrumente der } \\
\text { Kommunikation mit alten Freunden } \\
\text { - } \quad \text { Konflikte mit Eltern über die } \\
\text { Mediennutzung (Zeit, Kosten) } \\
\text { - } \quad \text { Probleme mit dem in den Medien } \\
\text { vermittelten Schönheitsideal } \\
\end{array}$ \\
\hline 5. Mario & $\begin{array}{l}\text { Protokoll eines Gesprächs } \\
\text { zwischen } E L I Z A \text { und } \\
\text { Mario über das Spielen } \\
\text { von Gewaltcomputer- } \\
\text { spielen }\end{array}$ & $\begin{array}{l}\text { - Negative Wirkungen von Gewalt in } \\
\text { Computerspielen } \\
\text { - Gründe der Faszination von Gewalt } \\
\text { in den Medien } \\
\text { - Umgang mit vertraulichen } \\
\text { Informationen }\end{array}$ \\
\hline
\end{tabular}


Tabelle 10 (fortgesetzt)

\begin{tabular}{lll} 
Kapitel & Inhalt & Angesprochene Themen \\
\hline 6. Notbremse & ELIZA wird größenwahn- & - Konsequenzen aus bedenklichem \\
sinnig und dringt in Belas & Mediennutzungsverhalten \\
Computer ein & - Die Rolle der Eltern in der Medien- \\
& & erziehung \\
& & ,Denkstrukturen“ von Computern \\
& & vs. Denkstrukturen von Menschen \\
& & Sicherheit im Internet und Schutz \\
& & vor unerwünschten Übergriffen \\
\hline 7. Abschied & Belas letztes Gespräch mit & - Auflösung der Geschichte \\
& ELIZA. Es wird aufgelöst, & \\
dass Kapitel 3 bis 6 von & \\
Bela nur geträumt wurden & \\
\hline
\end{tabular}

Im Gegensatz zur ersten Unterrichtseinheit enthalten Die ELIZA-Protokolle keine klaren Handlungsanleitungen zum Umgang mit Alltagsmedien. Im Gegenteil: Der zunächst verständnisvolle Gesprächspartner ELIZA entpuppt sich zunehmend als kalt kalkulierende Maschine mit wenig Verständnis für den Hintergrund des Medienhandelns ihrer Gesprächpartner. In den die Lesegeschichte begleitenden Fragestellungen und Aufgaben (vgl. Anhang A13) werden die Kinder aufgefordert, Lösungen für die Probleme von Bela, Fiona und Mario zu finden, die den Betroffenen mehr helfen als die kurzsichtigen Lösungen des Computerprogramms ELIZA. Bei der Entwicklung neuer Lösungen können Schülerinnen, Schüler und Lehrkräfte auf das im Rahmen der ersten Unterrichtseinheit Vom Leichtmatrosen zum Medienlotsen erlernte Hintergrundwissen zurückgreifen.

\subsubsection{Konzeptevaluation und Durchführung}

Der Aufbau von Empathie zum Protagonisten der Geschichte und der Aufbau von Spannung über den Ausgang der Geschichte sind die zentralen Elemente, um die Kinder für Belas Probleme, seine Mediennutzung und deren Ursachen zu interessieren. Um zu gewährleisten, dass die Anlage der Geschichte und ihre erzählerische Umsetzung diesen Kriterien entspricht, wurden die Hintergründe der Unterrichtseinheit, das erste Konzept der Geschichte sowie Textbeispiele für ihre Umsetzung auf einem Lehrkräfteworkshop im Februar 2007 den am Unterrichtsprogramm teilnehmenden Lehrkräften vorgestellt. $\mathrm{N}=19$ Lehrkräfte der 20 
Unterrichtsklassen nahmen an dem Workshop teil, eine Lehrkraft fehlte ohne Angabe von Gründen ${ }^{169}$. Das auf dem Workshop vorgestellte Konzept erhielt bereits alle Elemente der fertigen Geschichte, es fehlten allerdings noch die Gesprächsprotokolle mit den anderen Jugendlichen (Kapitel 4 „Fiona“ und Kapitel 5 „Mario). Obwohl die Textbeispiele und die Anlage der Lesegeschichte großen Anklang bei allen anwesenden Lehrkräften fanden, wurde von mehreren Lehrkräften der Wunsch geäußert, außer der Geschichte von Bela noch weitere thematische Ankerpunkte zu setzen, um weitere Problemfelder der kindlichen und jugendlichen Mediennutzung bearbeiten zu können. Aus diesem Grund wurden die Gesprächsprotokolle mit Fiona und Marion eingefügt, in denen die Themen Chatten und SMSen, Mediengewalt und medienvermittelte Schönheitsideale verarbeitet wurden. Außerdem wurde von einer Lehrkraft die Befürchtung geäußert, eine zu spannende beziehungsweise ,gruselige“ Geschichte könne bei Kindern oder bei den Eltern auf Ablehnung stoßen. Aufgrund dieser Anmerkung wurde der Traumcharakter der Geschichte stärker herausgestellt.

Darüber hinaus wurde im Rahmen des Workshops über weitere Möglichkeiten der Elterninformation debattiert. Von mehreren Lehrkräfte wurde dabei darauf verwiesen, dass ihnen im Anschluss an die letzten Weihnachtsferien aufgefallen sei, wie stark zu Weihnachten geschenkte Video- und Computerspiele, Fernseher und Spielkonsolen das Medienverhalten ihrer Schülerinnen und Schüler in Richtung einer inhaltlich und zeitlich problematischeren Nutzung beeinflusst hätten. Obwohl im Rahmen des Workshops nicht verifiziert werden konnte, inwieweit die beschriebenen Fallbeispiele auch einen empirisch nachweisbaren Trend beschreiben, wurde mit den Lehrkräften vereinbart, den Eltern vor dem nächsten Weihnachtsfest eine weitere Informationsbroschüre zukommen zu lassen. Kritik wurde auch an der zu erwartenden zeitlichen Dauer der Unterrichtseinheit laut, die in etwa den gleichen zeitlichen Rahmen wie die erste Unterrichtseinheit benötigen sollte. Da in sieben Unterrichtsklassen nach den Sommerferien die Klassenlehrkraft gewechselt hatte, mussten einige der „neuen“ Lehrkräfte überzeugt werden, die Unterrichtseinheit in ganzer Länge durchzuführen, obwohl sie selbst nie einer Durchführung dieser Einheiten zugestimmt hatten. Zwei neu hinzugekommene Lehrkräfte ließen sich überzeugen, bei zu starker Zeitknappheit die Unterlagen zum Unterricht an andere in der Klasse

169 Dabei handelte es sich um die Lehrkraft der Klasse, in der bereits ein Jahr zuvor kein Unterricht durchgeführt worden war. Nachträgliche Recherchen ergaben, dass auch in dieser Klasse nach dem Sommer die Klassenlehrkraft gewechselt hatte und offenbar alle betreffenden Unterlagen sowie die Einladung zum aktuellen Workshop nicht an die neue Klassenlehrkraft weitergegeben worden waren. 
unterrichtende Kolleginnen oder Kollegen weiterzugeben, so dass diese die Durchführung des Unterrichts übernehmen. Da im Rahmen des Lehrkräfteworkshops deutlich wurde, dass in den meisten anderen Unterrichtsklassen nach der vierten Klasse ebenfalls ein Wechsel der Klassenlehrkraft stattfinden würde, wurden die derzeitigen Klassenlehrerinnen und Lehrer dringend gebeten, ihren Nachfolgerinnen beziehungsweise Nachfolgern möglichst alle Informationen und Unterrichtsmaterialien zum Medienunterrichtsprogramm zu übergeben, um auch eine dritte Unterrichtseinheit in der fünften Klasse möglich zu machen. Allerdings wurde von mehreren Lehrkräften deutliche Skepsis dahingehend geäußert, ob eine weitere Unterrichtseinheit im folgenden Jahr, ob nun von ihnen oder einer Nachfolgelehrkraft verantwortet, Platz im dichten Lehrplan der fünften Klasse finden würde.

Nach einer auf den Lehrkräfteworkshop folgenden zweiwöchigen Bearbeitung der Geschichte wurde allen 20 Unterrichtsklassen Anfang März 2007 die fertige Lesegeschichte inkl. Aufgabenbeispielen im Klassensatz zu Verfügung gestellt. In einer Klasse (derselben, in der bereits ein Jahr zuvor kein Unterricht stattfand), wurde kein Unterricht durchgeführt. In 19 der 20 Unterrichtsklassen wurde nach Angaben im Lehrkräftefragebogen Medienunterricht durchgeführt. In 17 Klassen wurde die Lesegeschichte Die ELIZA-Protokolle verwendet. Eine Lehrkraft befand die Geschichte als nicht angemessen für ihre Schülerschaft und entwickelte in Anlehnung an die Inhalte der ursprünglichen Geschichte eine eigene Geschichte. In einer weiteren Klasse wurde die Geschichte ebenfalls nicht verwendet, ohne dass Angaben darüber vorliegen, welche Inhalte stattdessen im Unterricht behandelt wurden. Aufgrund der fehlenden Angaben zu den alternativen Aktivitäten wird in der Folge davon ausgegangen, dass in dieser Klasse kein den ELIZA-Protokollen gleichwertiger Medienerziehungsunterricht stattfand. Dahingegen fand mit der Lehrkraft, die eine eigene Geschichte und eigene Aufgabenstellungen zu dieser Geschichte erarbeitet hatte, ein ausführlicher Austausch zu den Inhalten des modifizierten Unterrichts stattfand, so dass die selbst entwickelte Einheit dem ursprünglichen Konzept als so ähnlich eingeschätzt werden kann, dass die Durchführung der Unterrichtseinheit in dieser Klasse als gegeben bewertet wird. Damit wurde der Medienunterricht Die ELIZAProtokolle in 17 der 20 Unterrichtsklassen durchgeführt, in einer Klasse wurde eine vom Inhalt und Zeitaufwand vergleichbare Unterrichtseinheit durchgeführt. Im November des gleichen Jahres wurde den Lehrkräften aller 20 Unterrichtsklassen ein Klassenset von DVDs mit beiliegender dreisprachiger Informationsbroschüre zugesandt mit der Bitte, die DVDs über die Schülerinnen und Schüler an die Eltern weiterzugeben. Auf der DVD befand sich ein Beitrag der Fernsehsendung nano (ausgestrahlt auf dem Sender 3sat, Erstausstrahlung am 
10.09.2007) über das Berliner Medienunterrichtsprogramm. In der beiliegenden Informationsbroschüre wurden den Eltern aller Unterrichtsklassen auf deutsch, türkisch und russisch erste Erfolge ${ }^{170}$ der Unterrichtseinheit im Hinblick auf die Medienausstattung, die Mediennutzung und die schulische Leistung der Kinder in den Unterrichtsklassen dargestellt. Mithilfe dieser Materialien sollten Eltern in der Vorweihnachtszeit motiviert werden, ihren Kindern ausschließlich altersgemäße Medieninhalte zugänglich zu machen, Vereinbarungen über Mediennutzungszeiten zu treffen und keine Fernseher oder Spielkonsolen im Kinderzimmer zu erlauben.

\subsubsection{Evaluation der Programmdurchführung}

Analog zum Vorgehen bei der Evaluation der ersten Unterrichtseinheit wurde auch die Durchführung der zweiten Unterrichtseinheit durch eine Befragung der teilnehmenden Lehrkräfte evaluiert. Auch hier lag das Hauptaugenmerk auf der Identifikation möglicher Probleme in der Programmimplementation und der Erfassung von Sachverhalten, die möglicherweise intervenierende Größen im Rahmen der Programmwirksamkeitsevaluation darstellen.

Die Unterrichtseinheit wurde in den teilnehmenden Klassen zwischen April und Juni 2007 durchgeführt. Wie im Vorjahr fand die Befragung der $\mathrm{N}=20$ Lehrkräfte der Unterrichtsklassen im Rahmen des anstehenden Messzeitpunktes des Berliner Längsschnitt Medien statt (2007: t3). Von allen 19 Lehrkräften in denen Medienunterricht durchgeführt wurde (Die ELIZA-Protokolle beziehungsweise vergleichbarer Unterricht oder anderer Medienunterricht) wurden gültige Fragebögen ausgefüllt ${ }^{171}$. Von der Lehrkraft, in deren Klasse der Unterricht nicht durchgeführt worden war, lag zwar ein Fragebogen vor, dieser enthielt aber keine gültigen Angaben. Im Folgenden werden jeweils die Merkmale und Bewertungen aller 19 Lehrkräfte berichtet. Bei den Angaben zur Verwendung einzelner Unterrichtselemente werden nur die Angaben der 18 Lehrkräfte einbezogen, die Die ELIZA-Protokolle verwendeten.

170 Die in der Elternbroschüre berichteten Effekte wurden wissenschaftlich erstmals in Pfeiffer, Mößle, Kleimann \& Rehbein (2007) publiziert.

171 Fünf der befragten Lehrkräfte gaben an, den Unterricht nicht selbst durchgeführt zu haben bzw. in Kooperation mit anderen Lehrkräften. Da sie durch den Interviewer oder die Interviewerin instruiert worden waren, nicht zu beantwortende Fragen zum Unterricht mit der durchführenden Lehrkraft abzustimmen, wurden Angaben dieser Lehrkräfte, soweit sie vorlagen, dennoch als gültig angesehen. 
Die befragten Lehrkräfte waren durchschnittlich 46,9 Jahre alt (SD =7,1). 14 Lehrkräfte waren weiblich (73,7\%), fünf Lehrkräfte männlich. 12 Befragte kannten die Klasse, in der Medienunterricht durchgeführt wurde, ,sehr gut“, 7 gaben an, die Klasse „eher gut“ zu kennen (3-stufige Skala: 1 = „nicht gut“; $2=$,eher gut"; $3=$,sehr gut"). Alle Befragten gaben an, Klassenlehrerin beziehungsweise Klassenlehrer der Klasse zu sein. Im Schnitt wurde die jeweilige Klasse von den Befragten bereits seit 23,6 Monaten unterrichtet $(\mathrm{SD}=15,3)^{172}$. 16 Befragte unterrichteten das Fach Deutsch in der Klasse, 15 Befragte das Fach Sachkunde, 13 Lehrkräfte unterrichteten beide Fächer in der jeweiligen Klasse. Eine Lehrkraft unterrichtete keins der beiden Fächer (Hier wurde der Medienunterricht von einer anderen Lehrkraft durchgeführt, wobei nicht die Geschichte Die ELIZA-Protokolle verwendet wurde). Im Durchschnitt wurden für die Unterrichtseinheit Die ELIZA-Protokolle 11,1 Unterrichtsstunden benötigt ( $\mathrm{SD}=5,3$; Median: 10 Unterrichtsstunden). Die kürzeste Unterrichtseinheit dauerte 4 Unterrichtsstunden, die längste Einheit 20 Unterrichtsstunden. Damit fiel die für die zweite Unterrichtseinheit benötigte Unterrichtszeit um durchschnittlich drei Unterrichtsstunden kürzer aus als bei der ersten Unterrichtseinheit (vgl. S. 266).

Sechs der Befragten beurteilten den der Unterrichtseinheit vorausgehenden Workshop als „sehr gut“, elf befanden ihn als ,gut“, eine Person als „weniger gut", eine Person machte keine Angabe zu diesem Punkt (4-stufige Antwortskala: 1 = sehr gut; 2 = gut; 3 = weniger gut; 4 = gar nicht gut). Acht Lehrkräfte gaben an, die Lesegeschichte Die ELIZA-Protokolle sei „sehr gut“ bei den Kindern ihrer Klasse angekommen, in neun Klassen wurde die Geschichte nach Wahrnehmung der Lehrkräfte als „gut“ beurteilt (4-stufige Antwortskala: $1=$ sehr gut; 2 =gut; 3 = weniger gut; 4 =gar nicht gut), zwei Lehrkräfte machten keine gültige ${ }^{173}$ Angabe. Damit war die Akzeptanz der Geschichte in der Klasse deutlich positiv, aber nicht ganz so positiv wie die erste Unterrichtseinheit. Zu erklären ist dieser Punkt möglicherweise mit der Wahrnehmung der Lehrkräfte zur Altersangemessenheit der Lesegeschichte. Zwar wurde die Geschichte von 13 Lehrkräften als altersangemessen beurteilt, 5 Lehrkräfte (darunter auch jene Lehrkraft, die eine eigene Geschichte geschrieben hatte) gaben aber an, die Geschichte sei eher etwas für etwas ältere $\operatorname{Kinder}^{174}$ (eine

172 Die im Vergleich zur Vorjahrbefragung (t2; 2006: Durchschnittliche Unterrichtsdauer der Lehrkraft in der Klasse: 27 Monate; SD = 11,3) ist mit dem Wechsel der Klassenlehrkraft in 6 der erfassten Klassen nach den letzten Sommerferien zu erklären.

173 Dabei handelte es sich um diejenigen Lehrkräfte, in deren Unterricht statt der ELIZAProtokolle andere Inhalte verwendet wurden.

174 Konkrete Fragestellung: „Ist die Geschichte Die ELIZA-Protokolle dem Alter der Kinder in Ihrer Klasse angemessen?“ Es gab drei Antwortmöglichkeiten: 1.: „Ja, genau richtig.“ 
Lehrkraft machte keine Angabe). Bessere Bewertungen als in der ersten Unterrichtseinheit bekamen dagegen die vorbereiteten Aufgaben und Fragen. Neun Lehrkräfte beurteilten sie als „sehr hilfreich“, acht Lehrerinnen und Lehrer befanden sie als „eher hilfreich“, drei Befragte - darunter jene beiden, in deren Klassen andere Inhalte verwendet wurden - machten keine Angaben zu diesem Punkt (4-stufige Antwortskala: 1 = sehr hilfreich; 2 = eher hilfreich; 3 = weniger hilfreich; 4 = gar nicht hilfreich).

Auch die Angemessenheit der Unterrichtseinheit bezüglich der Handlungsfreiheit der Lehrkräfte ${ }^{175}$ wurde von den Befragten positiver beurteilt als bei der ersten Unterrichtseinheit: Hatten im letzten Jahr 14 Lehrkräfte geäußert, die Materialien seien „gerade richtig“, befanden dieses Mal alle 16 Lehrkräfte, die zu diesem Punkt eine Angabe machten, die Unterrichtsmaterialen als ,gerade richtig“, 3 Lehrkräfte - darunter jene beiden, in deren Klassen andere Inhalte verwendet wurden - machten keine Angaben zu diesem Punkt. Etwas positiver als beim letzten Mal (vgl. S. 266) äußerten sich die Befragten auch zu der Frage, inwieweit die Unterrichtsinhalte eine längerfristige Wirkung auf das Verhalten der Schülerinnen und Schüler habe (4-stufige Antwortskala: $1=$,ja, auf jeden Fall“; 2 = „eher ja“; 3 = ,eher nein; 4 = „,nein, auf keinen Fall“). Drei Lehrkräfte gaben an, es gäbe ,auf jeden Fall“ längerfristige Wirkungen, zehn Lehrkräfte urteilten mit „eher ja“, vier Lehrkräfte mit „eher nein“, zwei Lehrkräfte - jene beiden, in deren Klassen andere Inhalte verwendet wurden - machten keine Angaben.

Da im Rahmen der zweiten Unterrichtseinheit die Lesegeschichte sowie die zugehörigen Fragen und Aufgaben die einzigen verwendeten Unterrichtsmedien darstellen, wurden die Lehrkräfte gebeten, Angaben darüber zu machen, inwieweit alle Kapitel, Fragen und Aufgaben auch tatsächlich bearbeitet wurden. In Tabelle 11 sind die Antworten der Lehrkräfte auf diese Fragen dargestellt. Dabei zeigt sich, dass alle Kapitel der Geschichte von 16 Klassen bearbeitet wurden. Die Lehrkraft, von der keine detaillierten Angaben zur Verwendung einzelner Kapitel und Aufgaben gemacht werden konnten (vgl. Fußnote 176) gab an, zumindest Kenntnis darüber zu haben, dass die Geschichte im Unterricht

2.: „Nein, die Geschichte ist eher passend für jüngere Kinder.“ 3.: „Nein, die Geschichte ist eher passend für ältere Kinder.“

175 Konkret lautete die Frage: „Die Unterrichtseinheit ist so gestaltet, dass Sie relativ frei in der konkreten Gestaltung des Unterrichtes sind. Wie beurteilen Sie diesen Aspekt?“ Es gab drei Antwortmöglichkeiten: 1.: „Ich hätte mir mehr konkrete Vorschläge für die Planung und Gestaltung des Unterrichtes gewünscht.“ 2.: „Ich wäre in meinen Entscheidungen über Planung und Gestaltung des Unterrichtes gerne freier gewesen." 3.: „Ich fand die Vorschläge für Planung und Gestaltung des Unterrichtes gerade richtig.“ 
verwendet wurde. Auch die zugehörigen Fragen wurden ganz überwiegend im Unterricht bearbeitet, lediglich in drei Kapiteln gaben jeweils unterschiedliche Lehrkräfte an, die Fragen nicht bearbeitet zu haben. Am wenigsten Verwendung fanden die vorgeschlagenen Aufgaben, wobei aber auch hier insgesamt von einem guten Implementationsniveau der vorgeschlagenen Aufgaben gesprochen werden kann. Insgesamt spiegelt sich in der großen Akzeptanz der Fragen und Aufgaben die recht positive Einschätzung der Lehrkräfte zur Qualität der Fragen und Aufgaben wieder, die oben bereits berichtet wurde.

Tabelle 11: Verwendung der verschiedenen Elemente der Unterrichtseinheit $\left(\mathrm{N}=16^{176}\right)$

\begin{tabular}{|c|c|c|c|c|c|c|}
\hline & \multicolumn{2}{|c|}{ Kapitel gelesen } & \multicolumn{2}{|c|}{ Fragen bearbeitet } & \multicolumn{2}{|c|}{ Aufgaben bearbeitet } \\
\hline & $\begin{array}{l}\text { ganz oder } \\
\text { teilweise }\end{array}$ & nein & $\begin{array}{l}\text { ganz oder } \\
\text { teilweise }\end{array}$ & nein & $\begin{array}{l}\text { ganz oder } \\
\text { teilweise }\end{array}$ & nein \\
\hline 1. Nachtschicht & 16 & - & 16 & - & 12 & 4 \\
\hline $\begin{array}{l}\text { 2. Ein Gespräch } \\
\text { in der Dunkelheit }\end{array}$ & 16 & - & 15 & 1 & 14 & 2 \\
\hline $\begin{array}{l}\text { 3. Ich bin ein } \\
\text { Meister aller }\end{array}$ & 16 & - & 16 & - & 13 & 3 \\
\hline Waffen & & & & & & \\
\hline 4. Fiona & 16 & - & 16 & - & 15 & 1 \\
\hline 5. Mario & 16 & - & 15 & 1 & 14 & 2 \\
\hline 6. Notbremse & 16 & - & 15 & 1 & \multicolumn{2}{|c|}{ Keine Aufgabe } \\
\hline 7. Abschied & 16 & - & Keine & gen & 15 & 1 \\
\hline
\end{tabular}

Insgesamt ergibt sich hinsichtlich der Implementation und Durchführung der zweiten Unterrichtseinheit ein zweigeteiltes Bild. Einerseits konnte die Einheit lediglich in 17 von 20 Klassen in der ursprünglich konzipierten Form durchgeführt werden, in zwei Klassen wurden selbst entwickelte beziehungsweise nicht näher beschriebene Medienunterrichtskonzepte eingesetzt (wobei Inhalte und Länge des von einer Lehrkraft selbstentwickelten Unterrichts mit den ELIZA-Protokollen durchaus vergleichbar sind), in einer Klasse (derselben, die bereits das erste Unterrichtskonzept nicht angenommen hatte) wurde gar kein Medienunterricht durchgeführt. In den 17 Klassen, in denen Die ELIZA-

176 Berücksichtigt wurden Angaben von N $=16$ Lehrkräften, in deren Unterricht Die ELIZAProtokolle verwendet wurden. Eine Lehrkraft konnte keine Angaben über die Verwendung einzelner Elemente machen, da der Unterricht von einer anderen Lehrkraft durchgeführt wurde. 
Protokolle verwendet wurden, wurde das Material wiederum recht vollständig verwendet und stieß - nach Wahrnehmung der Lehrkräfte - auf durchaus gute Akzeptanz bei den Schülerinnen und Schülern. Dies wiederum ist als Implementationserfolg zu interpretieren. Allerdings wurde von mehreren Lehrerinnen und Lehrern kritisiert, die Lesegeschichte sei eher für ältere Schülerinnen beziehungsweise Schüler geeignet, was in einem Fall dazu führte, dass die Lehrkraft in ihrem Unterricht eine eigene Geschichte einsetzte. Das im Gegensatz zur ersten Unterrichtseinheit etwas „schlankere“ Konzept der ELIZAProtokolle führte dazu, dass etwas weniger Unterrichtzeit verwendet wurde als beim Konzept Vom Leichtmatrosen zum Medienlotsen. Auf der einen Seite ist dies im Hinblick auf die bessere Implementierbarkeit in anderen Schulklassen natürlich zu begrüßen. Auf der anderen Seite stellt sich aufgrund der zahlreichen Themenkomplexe der Unterrichtseinheit die Frage, inwieweit die Unterrichtsinhalte in teilweise weniger als zehn Unterrichtsstunden ausreichend zu bearbeiten waren. 


\subsubsection{Das dritte Unterrichtskonzept (5. Klassen)}

Mit den Unterrichtskonzepten Vom Leichtmatrosen zum Medienlotsen und Die ELIZA-Protokolle wurden Beispiele der Vermittlung medienerzieherischer Inhalte für dritte und vierte Grundschulklassen erarbeitet, mit denen Lehrkräfte und Grundschulkinder für die Wichtigkeit einer altersgerechten Mediennutzung sensibilisiert werden sollen und auf deren Grundlage Regeln für einen altersgerechten kindlichen Medienumgang im Grundschulalter erarbeitet werden können. Die Umsetzung des medienerzieherischen Unterrichts in Berlin eröffnete die Chance, die kindliche Alltagsmediennutzung ein weiteres Mal im selben Klassenverband zum Thema zu machen, da in Berlin die fünfte und sechste Klasse Teil der Grundschule sind. Da diese dritte Unterrichtseinheit zugleich die letzte Unterrichtseinheit war ${ }^{177}$, sollte sie einen abschließenden Impuls bei Schülerinnen und Schüler und deren sozialem Umfeld, Freunden und Familien, für die Entwicklung langfristig funktionaler Mediennutzungsmuster setzen. Gleichzeitig sollten die Schülerinnen und Schüler in die Lage versetzt werden, die in den letzten beiden Jahren behandelten Unterrichtsinhalte erneut aufzugreifen und kreativ zu verarbeiten. Damit sollte eine Idee des SMARTKonzeptes aufgegriffen werden, in dessen Rahmen die Schülerinnen und Schüler am Ende der Unterrichtseinheit (vgl. S. 214, Tabelle 2, Lektionen 14 -16) Möglichkeiten erarbeiteten, die gewonnenen Erkenntnisse vor anderen Schülerinnen und Schülern zu präsentieren und Kinder mit problematischer Mediennutzung zu motivieren, elektronischer Mediennutzung weniger Zeit in ihrem Alltag einzuräumen.

Vor diesem Hintergrund wurde die Idee entwickelt, alle beteiligten Klassen im Rahmen des Unterrichtes 10- bis 20-minütige Spielszenen entwickeln zu lassen, in denen sie auf Grundlage einiger vorgegebener Fallskizzen die problematischen Seiten der Nutzung elektronischer Medien darstellen, und Auswege aus problematischen Formen der Mediennutzung zeigen. Diese Szenen sollten in den Schulen vor Eltern, Lehrkräften und Kindern der dritten bis sechsten Klassen vorgeführt werden. Gleichzeitig sollte die Idee des SMARTProgramms aufgenommen werden, unter den am Medienunterricht beteiligten Schulklassen einen Wettbewerb auszutragen. Bestand der Wettbewerb im SMART-Programm darin, diejenige Klassengemeinschaft zu belohnen, die am

177 Auf diese Weise wurde gewährleistet, dass beim letzten Messzeitpunkt (t5) des Berliner Längsschnitt Medien am Ende der sechsten Klasse, ein Jahr nach der letzten Unterrichtsintervention, mögliche längerfristige Effekte des medienerzieherischen Unterrichts gemessen werden konnten. 
erfolgreichsten beim Schalt-aus!-Programm abgeschnitten hatte, sollte der Wettbewerb der Berliner Klassen in der kreativsten Umsetzung einer vorgegebenen Fallskizze bestehen. Da in rund der Hälfte der Unterrichtsklassen im fünften Schuljahr mutmaßlich ein Wechsel der Lehrkraft bevorstand, wurde entschieden, weder die Fallskizzen noch die genauen Modalitäten des Wettbewerbes genau festzulegen, sondern das genaue Vorgehen mit den beteiligten Lehrkräften abzustimmen, um eine möglichst hohe Teilnahmequote an der letzten Unterrichtseinheit zu gewährleisten. Zur Vorbereitung des Lehrkräfteworkshops im Februar 2008 wurden drei Fallskizzen entwickelt, in denen aktuelle Probleme kindlicher beziehungsweise jugendlicher Mediennutzung aufgegriffen wurden: das Mobbing in internetbasierten Schüler-Communities, die Nutzung äußerst gewalthaltiger Computerspiele in einer Clique männlicher Schüler und Computerspielabhängigkeit. Zudem wurde ein Plan zur Veranstaltung eines eintägigen Theaterwettbewerbes entworfen, in dessen Rahmen die Schülerschaft aller Unterrichtsklassen die von ihnen entwickelten Szenen vor Schülerinnen, Schülern und Lehrkräften der anderen Schulen und einer Jury präsentieren sollten.

\subsubsection{Konzeptevaluation}

Das Unterrichtskonzept wurde im Februar 2008 auf einem Lehrkräfteworkshop vorgestellt und mit den anwesenden Lehrkräften diskutiert. 16 Pädagogen nahmen an dem Workshop teil, vier Lehrkräfte fehlten aus Zeit- oder Krankheitsgründen. Im Vorfeld der Organisation des Workshops stellte sich heraus, dass nach den Sommerferien in weiteren acht Klassen die Klassenleitung gewechselt hatte, so dass in diesem dritten Unterrichtsjahr lediglich sechs Lehrkräfte dabei waren, die von Anfang an der Teilnahme am Medienunterricht zugestimmt hatten. Obwohl die Idee der Entwicklung kurzer szenischer Inszenierungen von problematischer Mediennutzung und Lösungswegen aus solchen Nutzungsmustern zunächst bei der Mehrzahl der Lehrkräfte auf Zustimmung traf, zeigte sich bei der konkreten Planung des Vorhabens schnell, dass die meisten Lehrkräfte nicht bereit waren, einen ähnlichen zeitlichen Aufwand zu betreiben wie in den Vorjahren. Das wurde auch damit begründet, dass die Klassenlehrerinnen und -lehrer in der fünften Klasse weniger Fächer in ihren Klassen unterrichteten, als in der dritten und vierten Klasse, dass weniger Klassenleiterinnen und -leiter das Fach Deutsch unterrichteten und das Fach Sachkunde, das bisher ebenfalls für die Vermittlung der medienerzieherischen Inhalte genutzt wurde, in der fünften Klasse durch andere Fächer ersetzt wird 
(i. d. R. Naturwissenschaften, Erdkunde, Geschichte). Zudem konnte kein gemeinsamer Termin für die Veranstaltung eines gemeinsamen Wettbewerbstages gefunden werden, selbst die Veranstaltung von zwei Wettbewerbstagen mit jeweils der Hälfte der Klassen war nicht möglich. Zweitens sahen sich einige Lehrkräfte aufgrund fehlender didaktischer Expertise nicht in der Lage, mit ihren Klassen szenische Darstellungen zu entwickeln, mit denen die Schülerinnen und Schüler bei einem Wettbewerb in Konkurrenz zu anderen Klassen bestehen können. Darüber hinaus zeigte sich rund die Hälfte der Anwesenden nicht einverstanden mit der Idee, die Schülerinnen und Schüler ihre Szenen anhand vorgegebener Fallskizzen entwickeln zu lassen. Vielmehr wurde angeregt, die Kinder sollten die Szenen aufgrund persönlicher Erfahrungen mit ihren Alltagsmedien entwickeln. Besonders der letzte Punkt stellte vor dem Hintergrund der Gesamtkonzeption des Medienerziehungsunterrichts ein Problem dar. Bereits auf den Workshops in den beiden Jahren zuvor war von einigen Lehrkräften immer wieder kritisiert worden, die in der Unterrichtseinheit angesprochenen Probleme seien in der Lebenswelt der meisten ihrer Schülerschaft von wenig Relevanz. Auch wenn diese Kritik zunächst verständlich erscheint - es wurde bereits deutlich gemacht, dass zeitlich, inhaltlich und insbesondere funktional problematische Mediennutzungsmuster keineswegs die Mehrheit der Kinder betreffen - ist es doch Kennzeichen der meisten risikoorientierten Konzepte der Primärprävention, dass das zu vermeidende Risikoverhalten in der Regel nur bei einer Minderheit einer Population entwickelt wird. Wenn Lebensweltorientierung des Unterrichtes aber bedeutet, dass die Thematisierung explizit risikobehafteten Verhaltens im Rahmen der Prävention stark eingeschränkt wird, droht der Präventionscharakter der Maßnahme insgesamt verloren zu gehen. Insofern ergab sich als Essenz des Lehrerworkshops ein gewisses Dilemma: Letztlich waren weniger als die Hälfte der Lehrkräfte bereit und in der Lage, die inhaltlichen und organisatorischen Aufwände, den das in den Workshop eingebrachte Konzept bedeutet hätte, mitzutragen. Ein für die Inhalts- und Gestaltungswünsche der Lehrkräfte offeneres Konzept bedeutete aber zwangsläufig deutlich eingeschränkte Möglichkeiten der Steuerung inhaltlicher und gestalterischer Lernprozesse.

\subsubsection{Modifikation der Konzeptidee und Durchführung}

Aufgrund der geringen Akzeptanz des in den Lehrkräfteworkshops eingebrachten dritten Unterrichtskonzeptes wurde auf der Grundlage des ersten Vorschlages ein offeneres Konzept entwickelt, das dennoch wichtige Kern- 
bestandteile des ursprünglichen Konzeptes enthalten sollte. So wurde die Beibehaltung des Wettbewerbselements als wichtig angesehen, um die Teilnahmemotivation der Kinder aufrecht zu erhalten. Als weiterer wichtiger Punkt sollte das Transferelement erhalten bleiben: Das Unterrichtskonzept sollte bisher Gelerntes erneut aufgreifen und von den Kindern fordern, bisher erarbeitete Erkenntnisse vor anderen Schülerinnen und Schülern, Eltern und Lehrkräften darzustellen. Daher wurde ein neues Konzept mit weniger konkreten inhaltlichen und gestalterischen Vorgaben erarbeitet, welches diese Elemente enthielt und von dem vermutet wurde, dass es bei den beteiligten Lehrkräften auf größere $\mathrm{Zu}$ stimmung stoßen würde: Der Wettbewerb Medienlotsen gesucht!. Allen 20 Medienunterrichtsklassen des Berliner Längsschnitt Medien wurden Ausschreibungen für den Wettbewerb Medienlotsen gesucht! zugesandt (vgl. Anhang A15). Als Preise wurden die Finanzierung eines Tages- oder Wochenendausfluges der Klasse und Medien für die Klassenbibliothek ausgeschrieben, die von der Projektleitung zur Verfügung gestellt wurden. Im Ausschreibungstext wurden die Schülerinnen und Schüler darauf aufmerksam gemacht, dass sie in den letzen beiden Jahren zu regelrechten „Medienexperten“ ausgebildet worden sind. Nun wurden sie aufgefordert, ihr in den letzten beiden Jahren angesammeltes Wissen über Medien, Mediennutzung und die Chancen und Gefahren elektronischer Mediennutzung an andere Kinder ihrer Schule weiterzugeben. Dabei bekamen sie die folgende Aufgabenstellung:

Tragt in der Klasse alles zusammen, was Ihr über Medien und ihre sinnvolle Nutzung gelernt habt. Sammelt alles, was Ihr über Fernsehen, Computerspiele, Spielkonsolen, Handys, das Internet und andere Medien wisst und was Ihr über Chancen und Gefahren dieser Medien gelernt habt. Ihr könnt auch einen Medienexperten einladen, der Euch mit weiteren Tipps und Informationen unterstützt. Überlegt dann gemeinsam, wie Ihr Euer Wissen an andere Schülerinnen und Schüler Eurer Schule weitergeben könnt. Setzt Eure Idee in die Tat um und dokumentiert Euer Projekt. Diese Dokumentation ist Euer Wettbewerbsbeitrag.

Bis zum Ende der Frist von Wettbewerbsbeiträgen wurden fünf Klassenbeiträge eingereicht. Der Siegerbeitrag bestand in einem Multimediaprojekt, in dem sich die Schülerinnen und Schüler einer Klasse mit dem Kampf gegen die Grüne Langeweile beschäftigten. Dabei zeigten sie anhand von Bildern, Collagen und digitalen Präsentationen, wie Langeweile erfolgreich bekämpft werden kann, ohne den Fernseher oder den Computer einzuschalten. Mit selbst gemalten Bildern zur Geschichte Vom fernsehverrückten Frank illustrierten sie außerdem die Geschichte eines Kindes, in dessen Kinderzimmer eines Tages ein Fernseher einzieht und das Kind, welches buchstäblich vor den Fernseher gefesselt wird, nicht mehr aus dem Zimmer lässt. Beiträge drei weiterer Klassen thematisierten das Problem der exzessiven Computernutzung anhand von selbstgedrehten 
Videofilmen. Eine Klasse führte eine Befragung zur Mediennutzung der Schülerschaft ihrer Grundschule durch, verglich diese Ergebnisse mit den Resultaten derselben Befragung, die an der Partnerschule im schottischen Glasgow durchgeführt wurden und veröffentlichte einen mehrseitigen Forschungsbericht in der Schülerzeitung. Außerdem führte die Klasse ein kurzes Theaterstück im Rahmen eines schulweiten Gauklerfestes auf, in dem sie mit Tanz- und Akrobatikeinlagen Freizeitalternativen zur Mediennutzung zeigten. Zudem stellten die Schülerinnen und Schüler in der Schülerzeitung auch Computerspiele vor, die Spielspaß mit Lernen, Musizieren oder Bewegung kombinieren.

\subsubsection{Evaluation der Programmdurchführung}

Bereits die geringe Quote von Wettbewerbsbeiträgen (lediglich 5 von 20 Klassen) zeigt einen unbefriedigenden Erfolg bei der Implementation der dritten Unterrichtseinheit in der Gruppe der Unterrichtsklassen. In der im Rahmen des vierten Messzeitpunktes des Berliner Längsschnitt Medien (2008: t4) durchgeführten Befragung der teilnehmenden Lehrkräfte zeigte sich, dass über die fünf Teilnehmerklassen hinaus noch drei weitere Klassen an einem Wettbewerbsbeitrag gearbeitet hatten. Da das zeitliche Engagement dieser Klassen entweder gar nicht quantifiziert wurde oder mit maximal vier Unterrichtsstunden angegeben wurde und von den Lehrkräften auch nicht näher beschrieben wurde, konnte das Engagement dieser Klassen letztlich nicht als Beteiligung an der dritten Unterrichtseinheit gewertet ${ }^{178}$ werden, so dass im Ergebnis die Teilnahme von fünf Klassen an der dritten Unterrichtseinheit konstatiert werden kann. Eine Lehrkraft, die am Unterrichtsprogramm teilnahm, war seit Beginn der Studie ab der dritten Klasse dabei, eine Lehrkraft war ab der vierten Klasse in den Medienerziehungsunterricht involviert, drei Lehrkräfte waren bei dieser Unterrichtseinheit zum ersten Mal dabei. Damit zeigt sich, dass neu hinzugekommene Lehrkräfte zwar im Rahmen des Workshops zur dritten Unterrichtseinheit deutliche Bedenken hinsichtlich des zeitlichen und organisatorischen Aufwandes des Medienunterrichts äußerten, sich letztlich aber keineswegs überdurchschnittlich der Durchführung der Unterrichtseinheit verweigerten. Vielmehr wird deutlich, dass von den sechs Lehrkräften, die bereits zwei Unterrichtseinheiten durchgeführt hatten (die also von Anfang an grundsätzlich der Teilnahme am Unterrichtsprojekt zugestimmt hatten), lediglich eine Lehrkraft bereit war, sich bei der

178 Damit unterscheidet sich die Ergebnisdarstellung in dieser Arbeit von den Angaben in Mößle et al. (2010), wo von acht teilnehmenden Klasse berichtet wurde. 
Durchführung einer dritten Einheit zu engagieren. Die durchschnittliche Unterrichtsdauer in den teilnehmenden fünf Klassen betrug 11,4 Stunden $(\mathrm{SD}=6,2)$, wobei eine Klasse lediglich vier Unterrichtsstunden für den Wettbewerbsbeitrag verwendete. Die Klasse, die beim Wettbewerb den ersten Patz belegte, benötigte 15 Unterrichtsstunden für die Erarbeitung ihres Projektes, am längsten (20 Unterrichtsstunden) beschäftigte sich jene Klasse mit dem Projekt, die sowohl eine Befragung zur Mediennutzung in der eigenen Schule und der Partnerschule durchführte und darüber hinaus ein kleines Theaterstück entwickelte.

Bei der Auswertung der Lehrkräftefragebögen aller 19 Klassen der Unterrichtsgruppe (aus einer Klasse lag kein Fragebogen vor) zeigte sich, dass 15 Klassenlehrkräfte ${ }^{179}$ der Unterrichtsgruppe weiblich waren (75\%), fünf waren männlich. Von den am Unterricht teilnehmenden Lehrkräften waren vier weiblich, eine männlich. Im Durchschnitt waren die Lehrkräfte der Unterrichtsgruppe 48,8 Jahre alt $(\mathrm{SD}=7,2)$. Tatsächlich unterrichteten nur noch 12 Klassenleiterinnen und -leiter das Fach Deutsch. Damit bestanden, verglichen mit den ersten beiden Unterrichtseinheiten, die bisher ungünstigsten Bedingungen zur Implementation des Unterrichts durch die Klassenlehrkraft. Von den Lehrkräften der sechs Klassen, die den Medienunterricht durchgeführt hatten, gaben vier unter anderem das Fach Deutsch, eine Lehrkraft, die lediglich Mathematik in ihrer Klasse unterrichtete, hatte auf den Beitrag lediglich vier Unterrichtsstunden verwendet. Eine Lehrkraft, die ebenfalls kein Deutsch unterrichtete, dafür aber unter anderem Mathematik und Kunst, hatte mit ihrer Klasse das Befragungsprojekt zur Mediennutzung in ihrer Schule und der Partnerschule in Glasgow durchgeführt und die Ergebnisse im Mathematikunterricht in Form von Tabellen und Grafiken aufbereitet.

Der Wechsel zahlreicher Klassenlehrkräfte in den Klassen der Unterrichtsgruppe zeigte sich auch in den Angaben der Lehrkräfte, wie viele Monate sie ihre Klasse bereits unterrichten. Im Durchschnitt gaben die Lehrkräfte an, ihre Klassen seit 23,1 Monaten zu unterrichten $(\mathrm{SD}=15,8)$. Damit hatte sich dieser Wert im Vorjahresvergleich noch einmal leicht verringert (vgl. S. 266 in dieser Arbeit), obwohl 12 Monate zwischen den Messzeitpunkten lagen. Die subjektive Einschätzung der Lehrkräfte darüber, wie gut sie ihre Klasse kennen, wies ebenfalls auf eine eher hohe Fluktuation der Klassenleitungen im letzten Jahr hin: Sechs Lehrkräfte gaben an, die Klasse, in welcher der Unterricht durchgeführt werden sollte, ,sehr gut“ zu kennen, zehn gaben an, die Klasse „,eher gut“ zu kennen, eine Lehrkraft sagte, sie kenne ihre Klasse „nicht gut“ (3-stufige Skala:

179 Es lagen zwar nur von 19 der 20 Lehrkräfte Fragebögen vor, das Geschlecht der Klassenlehrkräfte ließ sich aber den jeweils aktuellen Organisationsplänen entnehmen. 
$1=$,nicht gut"; $2=$,,eher gut"; $3=$,gut“). Von zwei Lehrkräften, die einen Fragebogen abgegeben hatten, lagen diesbezüglich keine Angaben vor. Da der Medienunterricht nur in 5 von 20 Unterrichtsklassen durchgeführt wurde, und nur von diesen Lehrkräften einige Angaben zu ihrer Bewertung der Wirksamkeit und Akzeptanz der Einheit gemacht wurden, erscheint es schwierig, diese Daten im Hinblick auf die Implementation des ursprünglich erarbeiteten Konzeptes zu interpretieren, da die Heterogenität der einzelnen Unterrichtsprojekte keine eindeutigen Schlüsse darauf zulässt, ob die Einheit aufgrund der Grundkonzeption, der konkreten Umsetzung in der Klasse oder aufgrund des Engagements der Lehrkräfte als angemessen beziehungsweise wirksam eingestuft wurde ${ }^{180}$.

Insgesamt muss festgestellt werden, dass die Implementation der letzten Unterrichtseinheit als unbefriedigend zu bezeichnen ist. Einerseits ist dies mit den im Vergleich zu den Vorjahren relativ ungünstigen schulischen Rahmenbedingungen zu erklären: Durch die Reduktion der den Klassenlehrkräften zur Verfügung stehenden Unterrichtszeit, durch den Wegfall des Sachkundeunterrichtes und durch den geringen Anteil an Klassenlehrkräften, die das Fach Deutsch unterrichteten. Die Änderung des Unterrichtskonzeptes auf der Grundlage der Kritik der Lehrkräfte im Vorbereitungsworkshop konnte nicht erreichen, dass der Unterricht in der Mehrzahl der Klassen durchgeführt wurde. Vielmehr zeigt sich, dass einer grundsätzlich positiven Einstellung gegenüber dem Ursprungkonzept von ungefähr der Hälfte der Lehrkräfte im Workshop (vgl. S. 287), eine vollständige Beteiligung von lediglich 5 Klassen am ausgeschriebenen Wettbewerb gegenübersteht. Zudem wurde durch die größeren inhaltlichen Freiheiten der Lehrkräfte bei der Gestaltung des Wettbewerbsbeitrages eine weniger klar risikopräventive Ausrichtung des Unterrichts in Kauf genommen. So enthielt das ursprüngliche Konzept das bindende Element einer Aufführung/Präsentation des Wettbewerbsbeitrags vor der Schulöffentlichkeit und den Eltern, wodurch eine noch stärkere Sensibilisierung der Peergroup und

180 Auf die Frage, wie gut die Unterrichtseinheit bei den Schüler/inne/n angekommen sei, antwortete eine Lehrkraft mit „sehr gut“, 2 mit „gut“, 2 Lehrkräfte machten keine Angaben zu diesem Punkt. Als altersangemessen bewerteten 3 Lehrkräfte das Unterrichtskonzept, 2 gaben an, es sei eher für ältere Kinder angemessen. Hinsichtlich der Frage, ob die Lehrkräfte sich mehr konkrete Vorschläge für die Durchführung des Konzeptes gewünscht hätten, oder ob sie gerne noch freier bei der Gestaltung des Unterrichts gewesen wären, antworten 2 Lehrkräfte, sie wären gerne noch freier in ihren Gestaltungsmöglichkeiten gewesen, eine Lehrkraft fand die Anregungen durch das Konzept gerade richtig, eine Person hätte sich mehr konkrete Vorschläge gewünscht. Auf die Frage nach einer längerfristigen Wirkung des Unterrichtsprogramms antwortete eine Lehrkraft, die Einheit habe ,,auf jeden Fall“ eine längerfristige Wirkung, 4 Lehrkräfte antworteten mit „eher ja“. (Zur konkreten Fragestellung und Skalierung der hier berichteten Items vgl. S. 283). 
der Eltern erzielt werden sollte. Dieses Ziel konnte aufgrund der geringen Teilnahme der Unterrichtsklassen am Wettbewerb nicht erreicht werden. Dennoch muss festgehalten werden, dass die Wettbewerbsbeiträge der Klassen, die am dritten Unterrichtskonzept teilnahmen, eindeutig belegen, dass in diesen Klassen die Auseinandersetzung mit dem Thema der kindlichen Alltagsmediennutzung in der dritten Unterrichtseinheit und auch in den vorangehenden Einheiten sehr intensiv und kreativ verlief, so dass zumindest in diesen Klassen tatsächlich längerfristige Wirkungen des Unterrichtskonzeptes erhofft werden können.

\subsubsection{Fazit zur Implementation des dreistufigen Unterrichtsprogramms}

Die Implementation der Unterrichtseinheit zur Prävention problematischer Mediennutzungsmuster im Grundschulalter im Rahmen des Berliner Längsschnitt Medien verlief insgesamt recht erfolgreich. Das in Kapitel 8 formulierte allgemeine Implementationsziel konnte - trotz bestimmter Einschränkungen - in einem insgesamt zufriedenstellenden Maß erreicht werden. Das Unterrichtsprogramm zur Prävention problematischer Mediennutzungsmuster wurde von zufällig ausgewählten Grundschullehrkräften ab der dritten Klasse im Rahmen des regulären Deutsch- beziehungsweise Sachkundeunterrichts umgesetzt. Allerdings wurde der Medienunterricht nicht in allen ausgewählten Klassen durchgeführt, ferner kamen im Rahmen des Unterrichts nicht immer alle Bestandteile des Unterrichtskonzeptes zum Einsatz.

Anhand der auf Seite 229 formulierten Teilziele soll die Qualität der Gesamtimplementation des Unterrichtsprogramms zusammengefasst werden:

\section{(1) Die Lehrkräfte erklären sich nach einer offenen Diskussion über Art, Umfang und Wirkpotential des Unterrichtskonzeptes bereit, den Unter- richt durchzuführen.}

Während die erste Unterrichtseinheit von nach dem Lehrkräfteworkshop in allen zufällig ausgewählten Pretest-Klassen in Oldenburg und Soltau-Fallingbostel und in 19 der 20 zufällig ausgewählten Berliner Klassen ganz oder wenigstens teilweise durchgeführt wurde und die zweite Unterrichtseinheit in 18 Klassen durchgeführt wurde ${ }^{181}$, war die Implementationsquote bei der letzten Unterrichtseinheit (5 von 20 Klassen) unbefriedigend. Insgesamt zeigte sich, dass die

181 In 17 Klassen mithilfe des Originalmaterials (Die ELIZA-Protokolle), in einer Klasse mithilfe einer inhaltlich analogen, selbstentwickelten Geschichte. 
Konzeption der ersten beiden Unterrichtseinheiten den Bedürfnissen von Grundschullehrkräften in der dritten und vierten Klasse und den organisationalen Rahmenbedingungen in diesen Jahrgängen deutlich besser angepasst war, als den Besonderheiten Berliner Grundschulen in der fünften Klasse (häufiger Wechsel der Klassenlehrkraft nach der vierten Klasse, weniger Unterrichtszeit der Klassenlehrkraft in der Klasse, Wegfall des Sachunterrichts, weniger Deutschlehrkräfte unter den Klassenlehrerinnen und -lehrern). Die Ausgangsüberlegung, mithilfe des (recht konventionellen) Instrumentes einer Lesegeschichte Kinder und Lehrkräfte an das Thema und die Problemstellungen kindlicher Alltagsmediennutzung heranzuführen, erwies sich sowohl bei der Konzeptevaluation im Rahmen der Vorbereitungsworkshops als auch bei der Durchführungsevaluation als geeigneter Zugang. Auch der Zugang zu den Klassen über die Klassenlehrkraft erwies sich in den ersten beiden Unterrichtsjahren als der richtige Weg, da die Fächer Deutsch und/oder Sachkunde in der weit überwiegenden Zahl der Fälle von diesen Lehrkräften unterrichtet wurden. Als nur eingeschränkt erfolgreich kann die Ansprache der Eltern durch das Unterrichtskonzept bezeichnet werden. Dadurch, dass nur wenige Klassen der Unterrichtsgruppe an der dritten Unterrichtseinheit teilnahmen (und hier nur eine Klasse eine Schulaufführung umsetzte, die sich explizit auch an die Eltern richtete), konnten die vorher eingesetzten Elemente (Elternabende, Informationsbroschüre, Informations-DVD und Begleitbroschüre) durch die Aktivitäten der Schülerinnen und Schüler im Rahmen der dritten Unterrichtseinheit nicht weiter ergänzt werden.

Insgesamt wurde auch deutlich, dass ein kontinuierliches, über mehrere Jahre hinweg laufendes schulisches Medienerziehungsprogramm ohne eine verbindliche Verankerung im Lehrplan nur mit relativ großem Organisationsaufwand möglich ist. In jedem Lehrkräfteworkshop mussten einige Lehrerinnen und Lehrer erneut motiviert werden, an der Unterrichtseinheit teilzunehmen, die Wechsel der Klassenleitung machten es notwendig, immer wieder neue Lehrkräfte in das Programm einzubinden und auf einen ähnlichen Wissensstand zu bringen wie ihre Kolleginnen und Kollegen. Andererseits zeigten auch viele der Lehrkräfte, die von der ersten bis zur dritten Unterrichtseinheit an den Workshops teilnahmen, im dritten Unterrichtsjahr eine gewisse „Teilnahmemüdigkeit"،. Einen Anteil an der zuletzt eher geringen Teilnahmemotivation dieser Lehrkräfte hatte die Tatsache, dass neben der zeitlichen und organisatorischen Belastung durch die Vorbereitung und Durchführung der Unterrichtseinheiten auch noch die jährlichen Testungen und Befragungen der Klassen hinzukamen (t1 - 4: Zwei Testtage á 2 Schulstunden, t5: Ein Testtag á 2 Schulstunden), die in der Regel in der Unterrichtszeit der Klassenlehrkraft durchgeführt wurden. 
Insgesamt ist die Implementation - auch vor dem Hintergrund des hohen methodischen Anspruchs an die externe Validität der Implementation des Unterrichtsprogramms - dennoch als erfolgreich zu bewerten. So fand die Auswahl der Unterrichtsklassen streng randomisiert statt, so dass Selbstselektionseffekte nahezu ausgeschlossen waren. Die Teilnahme an jeder Unterrichtseinheit war für die Lehrkraft freiwillig, eine Lehrkraft hatte keinerlei Sanktionen zu befürchten, wenn sie nicht (mehr) am Medienunterrichtsprogramm teilnahm. Dennoch konnten 19 beziehungsweise 18 Klassenlehrerinnen und -lehrer motiviert werden, an zwei Unterrichtseinheiten teilzunehmen. Insofern legen die Ergebnisse der Evaluation der Durchführung des Unterrichtsprogramms nahe, dass die auf Seite 230 beschriebenen Wirksamkeitserwartungen nicht wesentlich durch eine mangelhafte Implementation der Unterrichtseinheiten gefährdet wurden.

\section{(2) Das Unterrichtskonzept wird nach seiner Durchführung von den Lehr- kräften als wirkungsvoll im Hinblick auf seine Wirkungsziele beurteilt.}

Sowohl im Rahmen des Konzepttests in Soltau-Fallingbostel und Oldenburg als auch in Rahmen der Hauptuntersuchung in Berlin wurde der ersten Unterrichtseinheit von der überwiegenden Mehrzahl der Lehrkräfte potentielle Wirksamkeit bescheinigt (vgl. S. 261 beziehungsweise 283). Auch die zweite Unterrichtseinheit wurde von rund drei Viertel der teilnehmenden Lehrkräfte als potentiell wirksam in Hinblick auf die Unterrichtsziele beschrieben (vgl. S. 283). Im Hinblick auf die dritte Unterrichtseinheit lässt sich trotz der insgesamt unbefriedigenden Implementationsquote immerhin festhalten, dass diejenigen Lehrkräfte, deren Klassen am Wettbewerb teilnahmen, den Unterricht für wirkungsvoll erachteten (vgl. S. 292, Fußnote 180). Insofern ist die Beurteilung der Lehrkräfte zur Wirksamkeit des Unterrichtsprogramms insgesamt als positiv zu beschreiben.

\section{(3) Das Unterrichtskonzept wird nach seiner Durchführung von den Lehr- kräften als dem Alter der Schülerinnen und Schüler angemessen be- urteilt.}

Zur Frage der Altersangemessenheit der drei Unterrichtseinheiten zeigte sich, dass alle drei Unterrichtseinheiten mehrheitlich als dem Alter der Kinder angemessen bezeichnet wurden, wobei ein Trend dahingehend zu erkennen ist, dass die erste Unterrichtseinheit die beste Bewertung erhielt und die dritte Unterrichtseinheit am schlechtesten bewertet wurde. So wurde die erste Unterrichtseinheit sowohl im Konzepttest als auch in der Berliner Hauptstudie fast aus- 
schließlich als altersangemessen bezeichnet, während die zweite Unterrichtseinheit „nur“ von knapp drei Vierteln der antwortenden Lehrkräfte als altersangemessen eingeschätzt wurde und die dritte Unterrichtseinheit gerade noch von der Mehrheit der antwortenden Lehrkräfte als altersgerecht eingeschätzt wurde.

(4) Das Unterrichtskonzept wird nach seiner Durchführung von den Lehrkräften als hinreichend konkret beurteilt, ohne dass pädagogische Gestaltungsmöglichkeiten einzelner Elemente als $\mathrm{zu}$ gering beurteilt werden.

Die Balance zwischen Gestaltungsfreiheit und Konkretheit der Unterrichtsvorschläge wurde bei den ersten beiden Unterrichtseinheiten sowohl im Rahmen des Konzepttests in Soltau-Fallingbostel und Oldenburg als auch in der Berliner Hauptstudie als überwiegend ,genau richtig“ bezeichnet, wobei sich die Berliner Lehrkräfte bei der ersten Unterrichtseinheit nicht ganz so positiv äußersten wie die Lehrkräfte beim Konzepttest in Norddeutschland, während die Passung der zweiten Unterrichtseinheit durchgängig als ,genau richtig“ bewertet wurde. Bei der dritten Unterrichtseinheit zeichnete sich hingegen kein klares Meinungsbild $\mathrm{ab}$.

(5) Das Unterrichtskonzept wird von den Schülerinnen und Schülern beteiligter Klassen angenommen.

Auf die Frage, wie die jeweilige Unterrichtseinheit bei den Kindern angekommen sei, antworteten sowohl die am Konzepttest in Norddeutschland als auch in Berlin teilnehmenden Lehrkräfte bezüglich der ersten beiden Unterrichtseinheiten fast ausschließlich, der Unterricht sei „sehr gut“ oder „gut“ angekommen, lediglich eine Berliner Lehrkraft bescheinigte der ersten Unterrichtseinheit, dass sie „weniger gut“ angekommen sei. Zur dritten Unterrichtseinheit lagen lediglich drei Angaben zu diesem Punkt vor, auch hier wurde angegeben, die Unterrichtseinheit sei „sehr gut“ oder „gut“ angekommen.

Es zeigt sich, dass die Implementation des Unterrichtskonzeptes sowohl im Konzepttest als auch in der Hauptstudie erfolgreich war, dass aber die dritte Unterrichtseinheit sowohl beim Implementationsniveau als auch in der Beurteilung durch die Lehrkräfte recht deutlich gegenüber den ersten beiden Unterrichtseinheiten abfällt. 